\title{
Universal physics of the few-body system of two neutrons and one flavored meson
}

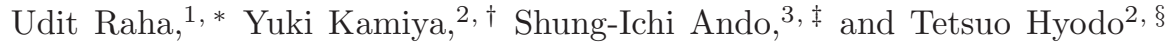 \\ ${ }^{1}$ Department of Physics, Indian Institute of Technology Guwahati, 781039 Assam, India \\ ${ }^{2}$ Yukawa Institute for Theoretical Physics, Kyoto University, Kyoto 606-8502, Japan \\ ${ }^{3}$ Department of Information Communication and Display Engineering, \\ Sunmoon University, Asan, Chungnam 31460, Korea
}

(Dated: September 11, 2018)

\begin{abstract}
We investigate the $s$-wave three-body system of two neutrons and one flavored meson with total spin-isospin $J=0, I=3 / 2$. The meson-neutron scattering length can become infinitely large when extrapolated to an unphysical region of the quark mass between strangeness and charm in the socalled zero coupling limit. Using a low-energy cluster effective field theory, we demonstrate that the Efimov effect is formally manifest in the three-body system when the meson-neutron scattering length approaches the unitary limit of the two-body interaction. We thereby discuss the consequence of remnant universal physics in the physical $K^{-} n n$ and $D^{0} n n$ systems.
\end{abstract}

\section{INTRODUCTION}

Few-body systems of an antikaon and nucleons are of great interest in the strangeness nuclear physics 1, 2]. The existence of the $\Lambda(1405)$ resonance below the $\bar{K} N$ threshold implies that the $\bar{K} N$ interaction in the isospin $I=0$ channel is strongly attractive with which the antikaon could be bound in nuclei 3, 44. Recently, threebody $\bar{K} N N$ systems have been a matter of intensive investigation based on rigorous few-body techniques [5-17]. To maximize the $I=0$ contribution of the $\bar{K} N$ pair in the $s$-wave $\bar{K} N N$ system, the state with the total spin $J=0$ and isospin $I=1 / 2$ is mainly studied. It is agreed among several different groups that the $J=0$ system supports a quasi-bound state below the threshold, although the quantitative predictions are not yet well converged. There are some studies of the state with $J=1$ and $I=1 / 2$, but the quasi-bound state is not found below the $\Lambda^{*} N$ threshold in Refs. [14, 18], presumably because of the small fraction of the $I=0$ component of the $\bar{K} N$ pair. In Ref. [12], it is shown that the $J=2$ and $I=3 / 2$ state is bound because of the $p$-wave $\bar{K} N$ interaction that generates the $\Sigma(1385)$ state.

Currently, nuclei with a heavy flavor (charm and bottom) meson is a subject of increasing attention [19]. This is triggered by the observation of many new heavy quarkonium-like hadronic states, so-called the $X Y Z$ states, above the open-charm or open-bottom thresholds [20 22]. Because the near-threshold states are often interpreted as hadronic molecular states, the existence of the $X Y Z$ states indicates that the heavy flavor meson could be a constituent in the formation of such exotic hadronic bound states. To assess the possible existence of the bound heavy mesons in nuclei, two-body $D N$ interactions [23 28] and three-body quasibound systems [29, 30] involving $D$ mesons have been

\footnotetext{
* udit.raha@iitg.ac.in

† yuki.kamiya@yukawa.kyoto-u.ac.jp

$\ddagger$ sando@sunmoon.ac.kr

$\S$ hyodo@yukawa.kyoto-u.ac.jp
}

studied extensively. With a plethora of high-precision data currently available from a host of modern experimental facilities, such as BaBar, Belle, CDF, D0, BES, $\mathrm{CMS}$, and $\mathrm{LHCb}$, similar investigations are also being extended to the open-bottom sector.

In general, the dynamics of a three-body problem reflect subtle details of two-body interactions. In some circumstances, gross properties of three-body systems can be assessed in terms of a few parameters which solely characterize the nature of two-body interactions. In fact, when the two-body scattering length $a$ is much larger than the interaction range $r_{0}$, microscopic details of twobody interactions become irrelevant as a consequence of low-energy universality [31, 32]. Universal physics can manifest themselves in sharply contrasting manner in the two- and three-body sectors. In the two-body sector, universal predictions are quite simple, manifested, e.g., as a shallow two-body bound state dimer for $a>0$ with the eigenenergy in the scaling limit given by $E_{\text {dimer }}=$ $-1 /\left(2 \mu_{\text {red }} a^{2}\right)$, where $\mu_{\text {red }}$ is the generic two-body reduced mass. However, universal predictions can become more complex in the three-body sector. The most striking phenomenon in a quantum mechanical three-body problem is the so-called Efimov effect [33], characterized by the emergence of an infinitely many and arbitrary shallow geometrically spaced bound states with accumulation point at zero energy in $s$-wave three-body systems. In the context of hadron physics, the Efimov effect and lowenergy universality have been discussed in three-nucleon systems 34 36], charmed meson systems 37], three-pion systems [38], and hyperon systems such as the $n n \Lambda$ [39].

In this work we have shed new light on the mesonnucleus systems having a strange (charm) quark, from the viewpoint of low-energy universality. We focus on the $\bar{K} N N(D N N)$ system with $J=0, I=3 / 2$, and $I_{3}=-3 / 2$, or more specifically, the $K^{-} n n\left(D^{0} n n\right)$ system. In these systems, all the two-body interactions occur in the $I=1$ combinations. In comparison to other quantum numbers, the quantum numbers associated with the $K^{-} n n\left(D^{0} n n\right)$ channel are ideal for examining the Efimov effect due to the following reasons. First, the absence of the Coulomb interaction guarantees that the 
low-energy behavior of this system is only governed by the two-body scattering lengths of the strong interaction. Second, the absence of any nearby coupled channels is also suitable for our purpose, otherwise the existence of such coupled channels is known to generally reduce the effective attraction in the three-body system [37, 38] diminishing the likelihood of formation of Efimov bound states.

While the scattering length of the two-neutron system is much larger than the typical length scale of the strong interaction, we should note that the magnitude of the physical $K^{-} n\left(D^{0} n\right)$ scattering length is not large enough for the meson-neutron two-body system to become resonant. However, our analysis reveals that the mesonneutron scattering length is expected to reach the unitary (resonant) limit, through an extrapolation of the quark mass from the strange sector to the charm, where the couplings to sub-threshold decay channels are eliminated. Consequently with such a zero coupling limit (ZCL), the otherwise complex meson-neutron scattering lengths turn out to be real valued. This kind of idealization obviously leads to a considerable simplification of the analysis of the three-body Faddeev-like integral equations compared to a more involved "realistic" analysis with complicated coupled-channel dynamics involving also hadrons other than the $K^{-}\left(D^{0}\right)$ meson and the neutron. Such a sophisticated approach is currently beyond the scope of our naive low-energy effective field theoretical (EFT) framework used in this work. Our primary objective in this paper is to present a simple leading order EFT analysis in an idealized limit to illuminate certain aspects of remnant two- and three-body universal physics that may be associated with bound state (dimer and trimer) formation in physical systems such as $K^{-} n\left(D^{0} n\right)$ and $K^{-} n n$ $\left(D^{0} n n\right)$. In principle, the universal features of unphysical quark mass system can be studied in lattice QCD simulations, as previously demonstrated in the context of few-nucleon systems [40].

This paper is organized as follows. In Sec.II, we study the two-body interaction of a flavored meson $\left(K^{-}\right.$or $\left.D^{0}\right)$ with a neutron in the $I=1$ state. We introduce a coupled-channel scattering model to perform an extrapolation from the strangeness sector to that of the charm. We evaluate the two-body scattering length in this extrapolation, together with the ZCL where the couplings to sub-threshold decay channels are artificially switched off. In Sec. III we formulate a pionless cluster effective field theory which describes the low-energy properties and dynamics of a three-particle cluster state consisting of two neutrons and a flavored meson $\left(K^{-}\right.$or $\left.D^{0}\right)$. In particular, we consider a possible scenario where the $s$-wave meson-neutron scattering length is infinitely large, while the $s$-wave neutron-neutron scattering length is fixed at its physical value. We perform an asymptotic analysis of the system of three-body integral equations to examine the renormalization group (RG) limit cycle behavior and other associated features related to the Efimov effect. Our numerical results for the full non-asymptotic analy- sis of the integral equations with and without including a three-body interaction are then presented. The final section is devoted to a summary of this work. Furthermore, based on our numerical results, a plausibility argument is presented at a qualitative level on the feasibility of $K^{-} n n$ or $D^{0} n n$ bound trimer formation. A discussion on certain numerical methodologies adopted in this work has been relegated to the appendices.

\section{TWO-BODY MESON-NEUTRON INTERACTION}

\section{A. $K^{-} n$ system, $D^{0} n$ system, and the unitary limit}

We first summarize the known properties of the mesonneutron interactions. In the $\bar{K} N$ system, several experimental data constrain the meson-baryon scattering amplitude [1]. Among others, the recent measurement of the kaonic hydrogen by the SIDDHARTA collaboration [41, 42] gives a strong constraint on the low-energy $\bar{K} N$ interaction, because the result is directly related to the $K^{-} p$ scattering length through the improved Desertype formula [43]. The analysis of the meson-baryon scattering with a complete next-to-leading order chiral SU(3) dynamics including the SIDDHARTA constraint [44, 45] determines the scattering lengths of the $K^{-} n$ system a: 1

$$
a_{0, K^{-} n}=-0.57_{-0.04}^{+0.21}-i 0.72_{-0.26}^{+0.41} \mathrm{fm} .
$$

The negative real part indicates that the $K^{-} n$ interaction is attractive, but not strong enough to support a quasibound state. The imaginary part of the scattering length indicates possible decay into sub-threshold $\pi \Sigma$ and $\pi \Lambda$ channels.

The $D^{0} n$ system is a counterpart of the $K^{-} n$ system in the charm sector. In contrast to the strangeness sector, there is no experimental data for the $D^{0} n$ scattering process. Theoretically, the $D^{0} n$ interaction has been studied by generalizing the established models in the strangeness sector [23 27] (see Ref. 19] for a recent review). Here we take an alternative strategy of using the experimental information of the $\Sigma_{c}(2800)$ resonance. The mass and width of the neutral $\Sigma_{c}^{0}(2800)$ state are given by the Particle Data Group (PDG) 22] as

$$
M_{\Sigma_{c}^{0}(2800)}=2806_{-7}^{+5} \mathrm{MeV}, \quad \Gamma_{\Sigma_{c}^{0}(2800)}=72_{-15}^{+22} \mathrm{MeV},
$$

which lies very close to the $D^{0} n$ threshold at $\sim 2803.8$ $\mathrm{MeV}$. Although the spin and parity of $\Sigma_{c}(2800)$ are not yet determined experimentally, by assuming $J^{P}=1 / 2^{-}$, we can determine the strength of the $s$-wave interaction

\footnotetext{
${ }^{1}$ In this paper, the scattering length is defined as $a_{0}=-f(E=0)$ with the scattering amplitude $f$. The sign convention of the scattering length is opposite to that used in Refs. [44, 45].
} 
in the $D^{0} n$ system. If the resonance pole of $\Sigma_{c}(2800)$ is found to lie below the $D^{0} n$ scattering threshold, then the $D^{0} n$ interaction is attractive enough to generate a quasi-bound state below the threshold. As will be demonstrated below, such a $D^{0} n$ quasi-bound picture of the $\Sigma_{c}(2800)$ resonance naturally arises in the $\mathrm{SU}(4)$ contact interaction model.

From these observations, we can draw the following conclusion regarding the meson-neutron interaction. On the one hand, the $K^{-} n$ system has a weakly attractive scattering length, as indicated by recent analysis in the strangeness sector. On the other hand, the $D^{0} n$ system can support a quasi-bound state below the threshold, which is ostensibly identified with the observed $\Sigma_{c}(2800)$ state. If we perform an extrapolation of the $K^{-} n$ interaction to the $D^{0} n$ interaction by changing the mass of the $s$ quark to that of the $c$-quark, we can expect the existence of an unphysical region of the quark mass between $m_{s}$ and $m_{c}$ where a very shallow quasi-bound state is formed when the magnitude of the scattering length becomes infinitely large. This represents the universal region around the unitary limit of the meson-neutron interaction. The situation is analogous to the two-nucleon [34], the twopion [38], and the $\Lambda \Lambda$ [46] systems, where the unitary limit of the two-hadron scattering is achieved by the quark mass extrapolation into the unphysical region. In much the same way we plan in this work to explore the universal physics in proximity to meson-neutron unitarity using unphysical quark masses between the strangeness and charm limits.

\section{B. Models of $\bar{K} N$ and $D N$ amplitudes}

To demonstrate that the unitary limit is indeed realized through the extrapolation, we employ a contact interaction model with flavor symmetry. In the strangeness sector, the Weinberg-Tomozawa model in the chiral SU(3) dynamics successfully describes the $\bar{K} N$ scattering [1, 47 49]. In the charm sector, the SU(4) generalization of this approach is developed in Ref. 24] in which the $\Lambda_{c}(2595)$ resonance in the $D N$ scattering is dynamically generated in the $I=0$ sector, analogous to $\Lambda(1405)$ in the strangeness sector. Evidently, there is one model in each sector which successfully describes the known experimental data. Therefore, in this work we describe both $\bar{K} N$ and $D N$ systems within a unified framework of a dynamical coupled-channel model. This enables us to perform the flavor extrapolation from strangeness to charm.

The coupled-channel scattering amplitude $T_{i j}(W)$ with the total energy $W$ is given by the scattering equation

$$
T(W)=\left[V^{-1}(W)-G(W)\right]^{-1},
$$

with the interaction kernel $V(W)$ :

$$
V_{i j}(W)=-\frac{C_{i j}}{f_{i} f_{j}}\left(2 W-M_{i}-M_{j}\right) \sqrt{\frac{M_{i}+E_{i}}{2 M_{i}}} \sqrt{\frac{M_{j}+E_{j}}{2 M_{j}}},
$$

where $C_{i j}$ is the coupling strength matrix specified below [see Eq. 10, $M_{i}\left(E_{i}\right)$ is the mass (energy) of the baryon in channel $i$, and $f_{i}$ is the channel-dependent meson decay constant. The loop function $G_{i}(W)$ has a logarithmic ultraviolet divergence which we tame employing dimensional regularization. After removal of the pole term through regularization, the finite constant parts proportional to $\ln (4 \pi)$, the Euler constant $\gamma_{E}$, etc., appearing in the loop function are then replaced by the subtraction constant $a_{i}\left(\mu_{\text {reg }}\right)$ at the regularization scale $\mu_{\text {reg. }}$. Such a term acts similar to a counter-term in a renormalizable theory (see, e.g., Refs. [49, 50]). Thus, we have

$$
\begin{aligned}
G_{i}\left(W ; a_{i}\right)= & \frac{2 M_{i}}{16 \pi^{2}}\left\{a_{i}\left(\mu_{\mathrm{reg}}\right)+\ln \frac{m_{i} M_{i}}{\mu_{\mathrm{reg}}^{2}}+\frac{M_{i}^{2}-m_{i}^{2}}{2 W^{2}} \ln \frac{M_{i}^{2}}{m_{i}^{2}}\right. \\
& +\frac{\bar{q}_{i}}{W}\left[\ln \left(W^{2}-\left(M_{i}^{2}-m_{i}^{2}\right)+2 W \bar{q}_{i}\right)\right. \\
& +\ln \left(W^{2}+\left(M_{i}^{2}-m_{i}^{2}\right)+2 W \bar{q}_{i}\right) \\
& -\ln \left(-W^{2}+\left(M_{i}^{2}-m_{i}^{2}\right)+2 W \bar{q}_{i}\right) \\
& \left.\left.-\ln \left(-W^{2}-\left(M_{i}^{2}-m_{i}^{2}\right)+2 W \bar{q}_{i}\right)\right]\right\}
\end{aligned}
$$

where $m_{i}$ denotes the mass of the meson in the channel $i$, and the quantity

$$
\bar{q}_{i}=\sqrt{\left[W^{2}-\left(M_{i}-m_{i}\right)^{2}\right]\left[W^{2}-\left(M_{i}+m_{i}\right)^{2}\right]}
$$

is the analytically continued three-momentum in the center-of-mass frame. In this model, the interaction strengths $C_{i j}$ are basically determined by the flavor symmetry. The free parameters in the model are the subtraction constants $a_{i}$. These real valued subtraction constants play the role of ultraviolet cutoffs, and effectively renormalize the dynamics of the channels which are not explicitly included in the model space. In this convention, the (diagonal) scattering length $a_{0, i}$ in channel $i$ is given by

$$
a_{0, i}=\frac{M_{i}}{4 \pi\left(M_{i}+m_{i}\right)} T_{i i}\left(M_{i}+m_{i}\right) .
$$

Next we consider the model space of the scattering. Because we are interested in the energy region near the $\bar{K} N / D N$ threshold, the channels with higher energies than the $\bar{K} N / D N$ thresholds (such as $\eta \Sigma / \eta \Sigma_{c}$ and $\left.K \Xi / K \Xi_{c}\right)$ are not very relevant to our analysis which we exclude 2 On the other hand, we include all relevant

2 By fitting the subtraction constants to experimental data, the contribution from the higher energy channels can be effectively renormalized. In fact, it is phenomenologically shown in Ref. [51] that the effect of the higher energy channels is not very strong around $\bar{K} N$ threshold energy region. 
channels lower than the $\bar{K} N$ and $D N$ thresholds, respectively, namely,

$$
K^{-} n, \quad \pi^{-} \Lambda, \quad \pi^{0} \Sigma^{-}, \quad \pi^{-} \Sigma^{0}
$$

in the strangeness sector, and the corresponding open channels in the charm sector:

$$
D^{0} n, \quad \pi^{-} \Lambda_{c}^{+}, \quad \pi^{0} \Sigma_{c}^{0}, \quad \pi^{-} \Sigma_{c}^{+} .
$$

Here the coupling matrix for these channels takes the form

$$
C=\left(\begin{array}{ccc}
1-\sqrt{\frac{3}{2}} \kappa & -\sqrt{\frac{1}{2}} \kappa & \sqrt{\frac{1}{2}} \kappa \\
0 & 0 & 0 \\
& 0 & -2 \\
& & 0
\end{array}\right) .
$$

A comment on the suppression factor $\kappa$ is in order. As discussed in Ref. 24], the microscopic origin of the contact interaction, Eq. (4), can be understood in terms of vector meson exchange picture. In this case, the interaction strength is proportional to $1 / m_{V}^{2}$ with $m_{V}$ being the mass of the exchanged vector meson. While $\mathrm{SU}(4)$ symmetry exactly requires $\kappa=1$, for the heavy flavor exchange processes, $C_{1 i}(i=2,3,4)$ should be significantly suppressed. Thus, as in Ref. 24], the suppression factor in the charm sector, $\kappa<1$, is introduced to account for the SU(4) breaking effects of the underlying mechanism. The masses of hadrons relevant for these channels are taken from the central values provided by the PDG [22], and for for convenience summarized in Table I We use the physical meson decay constants, $f_{\pi}=92.4 \mathrm{MeV}$ and $f_{K}=109.0 \mathrm{MeV}$, and the decay constant of the $D$ meson is also chosen to be $f_{D}=92.4 \mathrm{MeV}$, following Ref. 24]. The regularization scale is set at $\mu_{\text {reg }}=1 \mathrm{GeV}$, consistent with the typical scale of the vector mesons not explicitly incorporated in this framework 3

The remaining parameters are the subtraction constant $a_{i}$ in each channel and the suppression factor $\kappa$ in the coupling strength matrix, appearing in Eq. (10). To determine these parameters in the strangeness sector $\left(a_{i}^{s}\right.$ and $\left.\kappa^{s}\right)$, it is essential to take into account the SIDDHARTA data of the kaonic hydrogen [41, 42]. The data allows a direct extraction of the $K^{-} p$ scattering length as related through the improved Deser-type formula [4] and provides a strong constraint on the lowenergy $\bar{K} N$ interaction [44, 45]. In Ref. [45], a simplified model (termed as the "ETW" model) is constructed with the Weinberg-Tomozawa interaction $\left(\kappa_{s}=1\right)$ in the

3 It is important to note that the regularization scale can be chosen arbitrarily to suite the purpose of a given theory or model as long as the physical consequences are independent of this scale. This is, however, not to be confused with the hard or the breakdown scale of the same theory which in our context may be chosen as the pion mass, i.e., ${ }^{\star} \Lambda \sim m_{\pi}$, in commensurate with the pionless EFT analysis pursued in the three-body sector. model space of Eq. (8), but quite reasonably reproducing the full next-to-leading order amplitude constrained by the SIDDHARTA data. The explicit values of the subtraction constants $a_{i}^{s}$ in the ETW model are summarized in Table III The scattering length of the $K^{-} n$ system is obtained as

$$
a_{0, K^{-} n}=-0.135-i 0.410 \mathrm{fm} .
$$

Although the value deviates from the full result in Eq. (11) due to the simplified assumptions of this scattering model, both the order of magnitude and the qualitative features of the result (e.g., weak attraction) remain unchanged. We think this is sufficient for the purpose of our present analysis 4

In the charm sector, the subtraction constants $a_{i}^{c}$ were determined in Ref. 24] by the argument of "natural size" [49, 50]. However, a slight modification of the constant associated with the $D N$ channel was necessary to reproduce the observed $\Lambda_{c}^{+}(2595)$ resonance in the $I=0$ channel amplitude. Likewise, the same strategy is pursued in the present work for the $I=1$ two-body subsystem; the subtraction constants in channels $i=2-4$ (i.e., $\pi^{-} \Lambda_{c}^{+}, \pi^{0} \Sigma_{c}^{0}$ and $\left.\pi^{-} \Sigma_{c}^{+}\right)$are chosen to be -2.060 29, consistent with the "natural size" $a_{\text {nat }} \sim-2$ [49, 50] ${ }^{5}$ while the subtraction constant in channel 1 (i.e., for $D^{0} n$ ) is slightly adjusted to reproduce the $\Sigma_{c}(2800)$ state near the $D^{0} n$ threshold. Such fine-tuning of the value of the subtraction constant reflects further SU(4) breaking effects in our model. We also tune the value of $\kappa^{c}$ to control the width of the resonance. By choosing the subtraction constants $a_{i}^{c}$ shown in Table $\amalg$ with $\kappa^{c}=0.453$, we dynamically generate a resonance pole corresponding to the $\Sigma_{c}(2800)$ state at

$$
M=2800 \mathrm{MeV}, \quad \Gamma=72 \mathrm{MeV} .
$$

In this model, the $D^{0} n$ scattering length is found to be

$$
a_{0, D^{0} n}=0.764-i 0.615 \mathrm{fm} .
$$

The positive real part is in accordance with the existence of the quasi-bound state $\Sigma_{c}(2800)$ below the threshold.

\footnotetext{
4 We have checked that the central value given in Eq. (1) can be reproduced by adjusting $a_{1}^{s}=-0.649$ and $a_{2}^{s}=-1.899$. The qualitative conclusion in Sec. II, i.e., the emergence of the unitary limit between strangeness and charm sectors in the ZCL, remains unchanged with this parameter set. In this paper, we use the original subtraction constants of the ETW model [45], respecting the consistency with the $K^{-} p$ sector.

5 There are two arguments determining the natural size of the subtraction constants. In Ref. [49], through a matching of the loop function evaluated using dimensional and cut-off regularization methods, the natural size of these constants were estimated. In Ref. [50], the determination of the natural values of these constants was pursued utilizing the renormalization condition, $G_{i}\left(W=M_{i} ; a_{\text {nat }}\right)=0$, along with the constraints from the lowenergy behavior of the amplitudes and the physical requirements on the loop functions. In both cases, the natural value is estimated as $a_{\text {nat }} \sim-2$ at the regularization scale, $\mu_{\text {reg }}=1 \mathrm{GeV}$.
} 
TABLE I. Masses of hadrons.

\begin{tabular}{|c|c|c|c|c|c|c|c|c|c|c|c|}
\hline Hadron & $K^{-}$ & $\pi^{-}$ & $\pi^{0}$ & $n$ & $\Lambda$ & $\Sigma^{-}$ & $\Sigma^{0}$ & $D^{0}$ & $\Lambda_{c}^{+}$ & $\Sigma_{c}^{0}$ & $\Sigma_{c}^{+}$ \\
\hline Mass $[\mathrm{MeV}]$ & 493.677 & 139.57018 & 134.9766 & 939.565379 & 1115.683 & 1197.449 & 1192.642 & 1864.3 & 2286.46 & 2453.75 & 2452.9 \\
\hline
\end{tabular}

TABLE II. Subtraction constants $a_{i}^{s, c}\left(\mu_{\text {reg }}\right)$ at the regularization scale $\mu_{\text {reg }}=1 \mathrm{GeV}$.

\begin{tabular}{lcccc}
\hline \hline channel $i$ & 1 & 2 & 3 & 4 \\
\hline$a_{i}^{s}$ (strangeness) & -1.283 & 0.238 & -0.714 & -0.714 \\
$a_{i}^{c}$ (charm) & -1.663 & -2.060 & -2.060 & -2.060 \\
\hline \hline
\end{tabular}

The origin of the $I=1$ quasi-bound state can be understood in the following argument. Because of the $\mathrm{SU}(4)$ symmetry, the interaction of the $D^{0} n$ channel has the same sign as that of the $K^{-} n$ channel, while the strength at the threshold is enhanced by the ratio $m_{D} / m_{K}$. Thus, we can expect a stronger attractive interaction in the $D^{0} n$ channel. Moreover, the heavier reduced mass of the $D N$ system leads to suppression of the kinetic energy which is implicitly reflected by the larger value of the two-body scattering length. This is a crucial expedient in the manifestation of universality in the three-body $D^{0} n n$ system as demonstrated in the next section.

\section{Flavor extrapolation and zero coupling limit}

Next we introduce a parameter $0 \leq x \leq 1$ which controls the extrapolation from strangeness to charm. Assuming a linear relationship among the following model parameters, we vary each as a function of $x$;

$$
\begin{aligned}
m_{i}(x) & =m_{i}^{s}(1-x)+m_{i}^{c} x, \\
M_{i}(x) & =M_{i}^{s}(1-x)+M_{i}^{c} x, \\
a_{i}(x) & =a_{i}^{s}(1-x)+a_{i}^{c} x, \\
f_{i}(x) & =f_{i}^{s}(1-x)+f_{i}^{c} x, \\
\kappa(x) & =\kappa^{s}(1-x)+\kappa^{c} x,
\end{aligned}
$$

for the respective channels, $i=1, \ldots 4$. Here, $x=0(x=$ 1) corresponds to the physical point of the model in the strangeness (charm) sector, while all other intermediate values of $x$ represent the model in the unphysical domain. Thus, for instance, in the $i=1$ channel, by varying $x$ from 0 to 1 , we can perform a linear extrapolation from the $K^{-} n$ to the $D^{0} n$ scattering sectors.

Now we study the behavior of the complex scattering length in the coupled-channel contact interaction model. The real and imaginary parts of the meson-neutron scattering length as functions of $x$ are shown in Fig. 1 . The scattering length varies continuously from $a_{0, K^{-} n}$ to $a_{0, D^{0} n}$ along with $x$. It is not immediately clear from this figure how to decipher the remnant of the universal features of meson-neutron unitarity in any straightforward manner due to effects of the sub-threshold decay

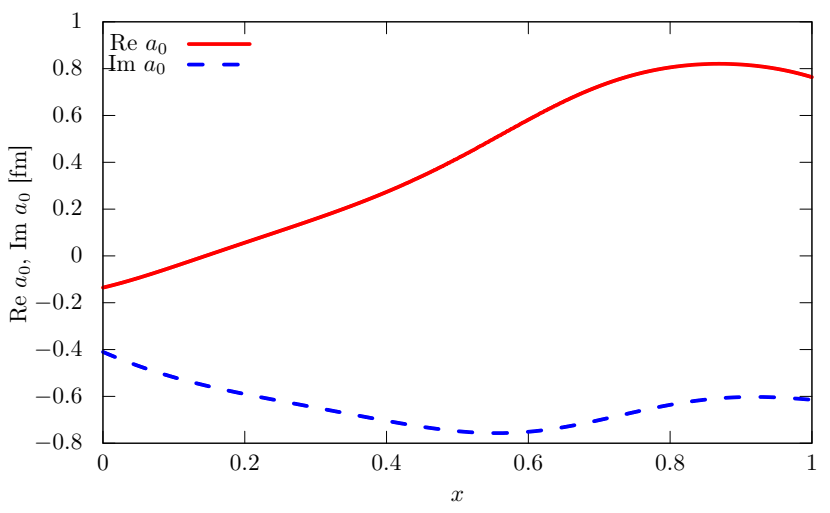

FIG. 1. Complex meson-neutron scattering length in the coupled-channel contact interaction model as a function of the extrapolation parameter $x$. Solid (dashed) line represents the real (imaginary) part.

channels. We note that in the present case, the $S$-matrix pole corresponding to the quasi-bound state of the $D^{0} n$ system moves to the higher positive energy region as we decrease $x$ from 1 , eventually going far above the $K^{-} n$ threshold at $x=0$ without yielding a quasi-bound $K^{-} n$ state. The pole trajectory of the scattering amplitude is shown in Fig. 2. Note that the pole is on the Riemann sheet with physical momentum with respect to channel 1 and unphysical momenta in regard to the others 6 Thus, the corresponding energy pole, $E_{h}=W-M_{1}(x)-m_{1}(x)$ (measured with respect to the threshold of the channel 1), directly influences the physical spectrum when Re $E_{h}<0$ (as in the charm sector, $x=1$ ), being on the most adjacent Riemann sheet to the physical real axis. On the other hand, its effect becomes less significant when $\operatorname{Re} E_{h}>0$ (as in the strangeness sector $x=0$ ), with the proximity of the pole position far away from the physical axis.

To elucidate a possible scenario to access the unitary limit of the meson-neutron interaction, we consider a zero coupling limit (ZCL) in which the channel couplings are

\footnotetext{
6 In an $n$-channel problem, the scattering amplitude is defined on a $2^{n}$-sheeted Riemann surface in the complex energy plane with momentum $p_{i}$ and $-p_{i}$ in channel $i$ corresponding to the same energy. The Riemann sheet is specified by choosing either the physical momentum ( $\operatorname{Im} p_{i}>0$, first sheet) or the unphysical momentum ( $\operatorname{Im} p_{i}<0$, unphysical sheet) for each channel $i$. The most adjacent Riemann sheet to the real scattering axis is obtained by choosing physical momenta in the open channels and unphysical momenta in the closed channels.
} 


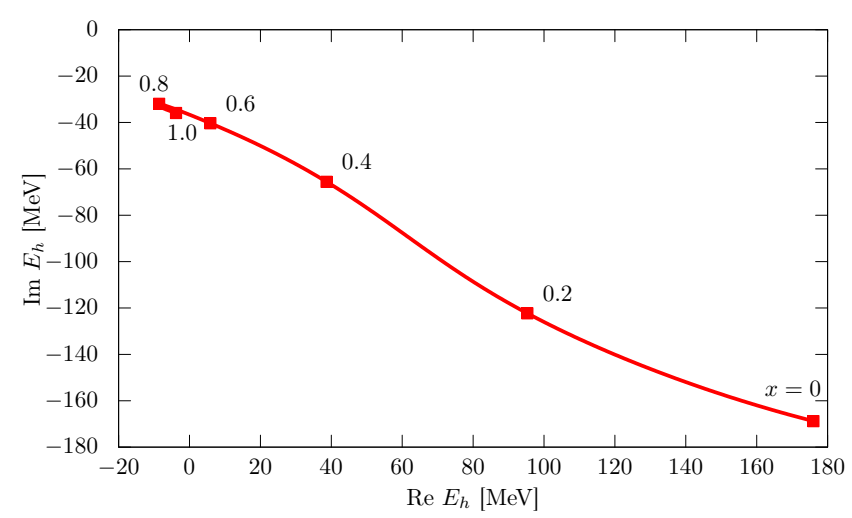

FIG. 2. Trajectory of the pole of the scattering amplitude in the coupled-channel contact interaction model. The energy is measured with respect to the threshold of channel 1, i.e., $E_{h}=W-M_{1}(x)-m_{1}(x)$.

artificially switched off [52 54], i.e.,

$$
C_{1 i}=C_{i 1}=0 \quad \text { for } i=2,3,4 .
$$

Under this assumption, the coupled-channel problem reduces to a single-channel scattering of the $K^{-} n / D^{0} n$ system. The $K^{-} n$ system is again found to have no bound state, while the previous quasi-bound state in the $D^{0} n$ channel now becomes a real bound state with invariant mass of $W \sim 2802 \mathrm{MeV}$. Correspondingly, the scattering length is real and negative (positive) in the $K^{-} n\left(D^{0} n\right)$ channel:

$$
a_{0, K^{-} n}^{\mathrm{ZCL}}=-0.394 \mathrm{fm}, \quad a_{0, D^{0} n}^{\mathrm{ZCL}}=4.141 \mathrm{fm} .
$$

The above numerical values of the scattering lengths in the ZCL strongly suggest that the meson-neutron interactions become resonant at an intermediate point in the unphysical domain $(0<x<1)$. In Fig. 3, we display the result for the inverse scattering length $1 / a_{0}(x)$, expressed as a function of the extrapolation parameter $x$. We indeed find that the unitary limit $\left(1 / a_{0}=0\right)$ is achieved at $x=0.615$. At this point, the extrapolated mass of the flavored meson in the channel 1 is

$$
m_{1}(0.615)=1336.61 \mathrm{MeV} \text {. }
$$

The corresponding behavior of the pole trajectory in the zero coupling limit is shown in Fig. 4. The real bound state pole at $x=1$ that lies on the physical (first) Riemann sheet turns into a virtual state pole at the unitary limit moving onto the unphysical Riemann sheet. As we further decrease $x$, the virtual state pole eventually meets with a second virtual state pole on the unphysical sheet and then acquires a finite width 55. In particular, at $x=0$, the virtual state pole remains still on the unphysical sheet with a finite imaginary part below the threshold.

As seen earlier the neglect of the decay channels in the ZCL yields real scattering lengths which may appear somewhat unrealistic in contrast to the full coupledchannel model that yields real and complex parts of the

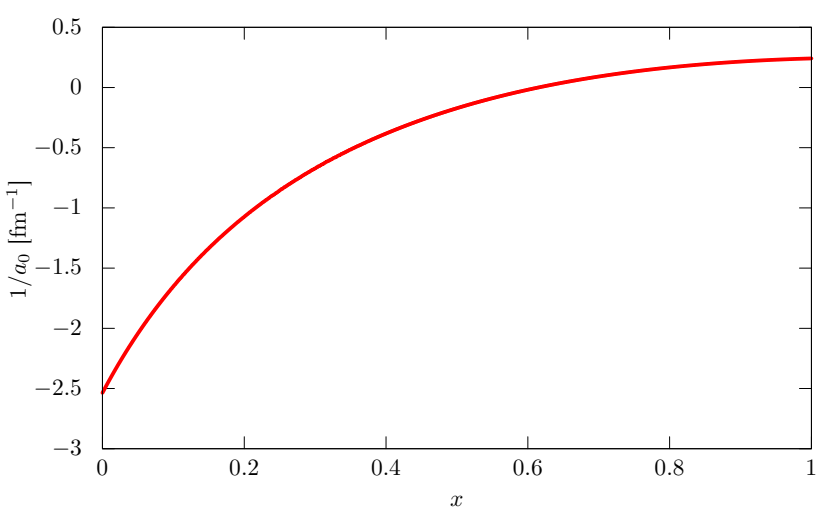

FIG. 3. Meson-neutron inverse scattering length in the absence of decay channels in the zero coupling limit as a function of the flavor extrapolation parameter $x$.

scattering lengths of roughly the same size. A natural question therefore arises as to the utility of the ZCL approach. To this end, let us make a comment to elucidate the significance of the ZCL analysis. One may think that the behavior of the scattering length (Fig. 3) and the pole trajectory (Fig. 4) in the ZCL are very much different from those in the full model (Figs. 11 and 2). However, the qualitative feature, namely, the existence of the pole remains unchanged by taking the ZCL. In fact, it is guaranteed by a topological invariant of the scattering amplitude that the existence of a pole is stable against the continuous change (deformation) of the model parameters, except for the special case where a zero of the amplitude exists near the pole (vide Ref. [56] for details). Because the ZCL can be achieved by gradually decreasing the off-diagonal couplings $C_{1 i}=C_{i 1}$, it is continuously connected with the full model. Hence, it is worth studying the model in the ZCL to check whether the system develops an eigenstate pole, although the exact position of the pole may show a large deviation. Furthermore, as we will show in the next subsection, the relation between the scattering lengths and the pole positions exhibits two-body universality, both in the full model and the model in the ZCL. Thus, the ZCL model and the full model share common features of universal physics in the two-body sector.

\section{Universality in the two-body sub-system}

It is instructive to take another look at the above results from the viewpoint of two-body universality. When the magnitude of the scattering length $\left|a_{0}\right|$ becomes large and approaches the unitary limit, universality suggests that there exists a two-body meson-neutron eigenstate with the eigenmomentum

$$
k_{a}=i / a_{0},
$$

up to corrections suppressed by effective range terms. This relation holds even for a complex value of $a_{0}$. Thus, 


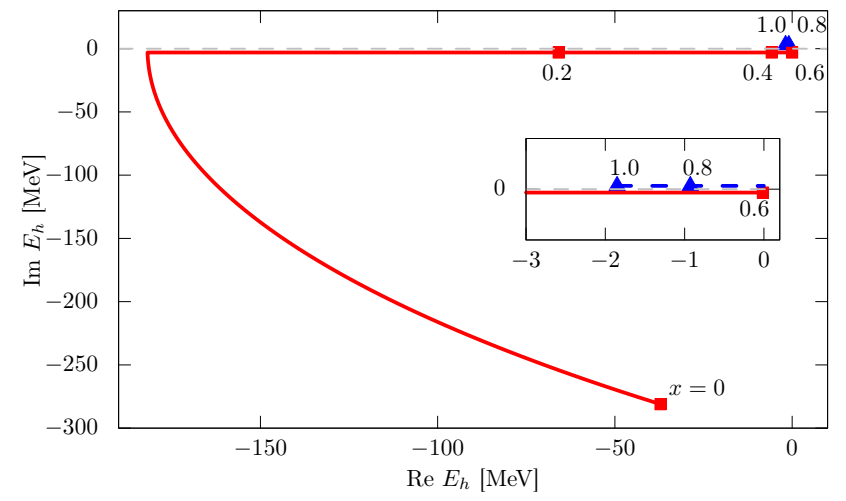

FIG. 4. Trajectory of the pole of the scattering amplitude in the zero coupling limit. The energy is measured with respect to the threshold of channel $1\left[E_{h}=W-M_{1}(x)-m_{1}(x)\right]$. Dashed (solid) line represents the pole on the physical (unphysical) Riemann sheet.

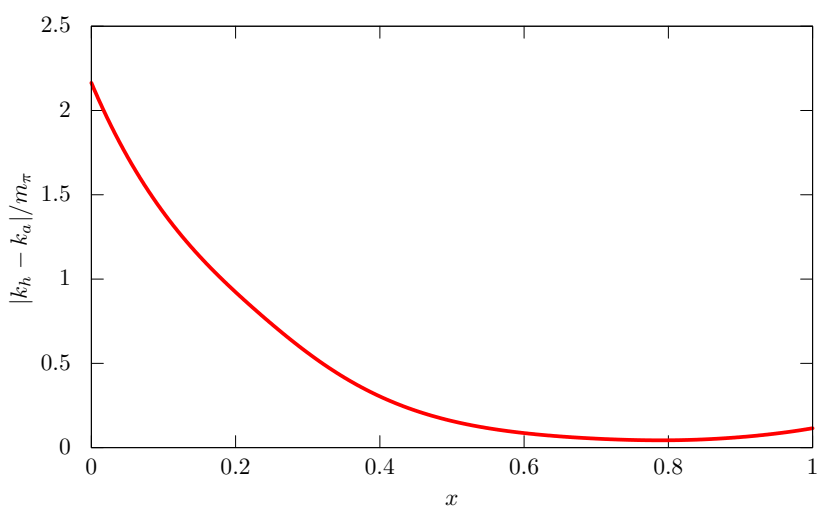

FIG. 5. Deviation of the eigenmomentum from the universal prediction $\left|k_{h}-k_{a}\right| / m_{\pi}$ as a function of the flavor extrapolation parameter $x$ in the coupled-channel contact interaction model.

if we calculate the eigenmomentum $k_{h}$, that corresponds to the pole position of the scattering amplitude in the system with a large scattering length, the true eigenvalue $k_{h}$ can be well approximated by $k_{a}$ in the two-body universal region. The deviation of $k_{h}$ from $k_{a}$ thus serves as a measure of the violation of universality.

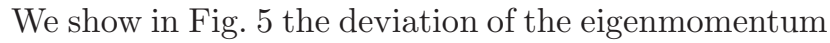
from the universal prediction $\left|k_{h}-k_{a}\right|$ normalized by the typical momentum scale of the strong interaction, i.e., the pion mass $m_{\pi}$, as a function of $x$. Although there is a large deviation in the strangeness sector $(x=0)$, the universal value $k_{a}$ around $x \sim 0.8$, including the $D^{0} n$ system at $x=1$, gives a reasonable prediction of the true eigenvalue $k_{h}$. Since the deviation is much smaller than the typical scale $m_{\pi}$, the result suggests that the full coupled-channel model, despite the influence of the decay channels, should also reflect the relevance of lowenergy universality in the meson-neutron system close to the charm quark sector, $x \sim 1$.

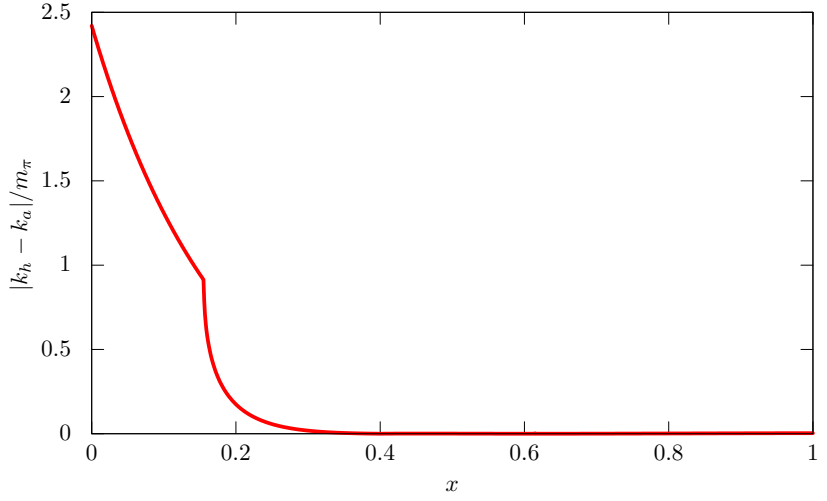

FIG. 6. Deviation of the eigenmomentum from the universal prediction $\left|k_{h}-k_{a}\right| / m_{\pi}$ in the zero coupling limit as a function of the flavor extrapolation parameter $x$.

The situation becomes much more conspicuous in the ZCL. As shown in Fig. 6, we find quite a good agreement of $k_{h}$ with $k_{a}$, not only in the vicinity of the unitary limit $(x \sim 0.6)$ but also in the entire region $x \gtrsim 0.3$. We should note that the eigenstate in the unbound region $(x \lesssim 0.6)$ corresponds to a virtual state $\left(a_{0}<0\right)$, an unphysical bound state with the S-matrix pole lying on the unphysical sheet. A characteristic cusp-like structure around $x \sim 0.15$ is caused when the virtual state acquires a finite width (also see Fig. (4).

Thus, our simplistic model approach demonstrates that universality is a powerful tool to investigate the meson-neutron two-body system, both in the full model and the ZCL.

\section{THREE-BODY SYSTEM OF TWO NEUTRONS AND ONE MESON}

\section{A. Effective field theory}

In this section, we consider the universal features in the three-body system consisting of two neutrons and one flavored meson with $J=0, I=3 / 2$, and $I_{3}=-3 / 2$, assuming that the two-body physics is finely tuned and characterized only by the two-body scattering lengths. A low-energy EFT can provide a simple but powerful systematic tool, for a quantitative analysis of such threebody systems in proximity to the scattering threshold. Recently, plethora of EFT based analyses have been used to predict the formation of bound states in a variety of three-body cluster states [39, 57 61] which are especially neutron-rich.

Primarily for the sake of a qualitative exploratory study of three-body universality, we henceforth neglect the sub-threshold decay channels $i=2-4$, which means that our three-body analysis is consistent with the ZCL idealization with real valued meson-neutron scattering lengths [see Eq. (20)]. Our motivation of this part of the 
study is to determine under what circumstance do we expect to find the three-body system to become bound solely by virtue of the Efimov "attraction". There is currently no consensus, either experimentally or theoretically, as to whether the system in the physical limits, $x=0$ (i.e., $\left.K^{-} n n\right)$ and $x=1\left(D^{0} n n\right)$, respectively, are bound. Through universality based arguments we hope to gain general insights alternative to rigorous realistic calculations which is beyond the scope of the present work.

Here we employ a leading order cluster effective field theory with a flavored meson $K^{-}\left(D^{0}\right)$ and a neutron $\psi_{n}$ as the elementary fields in the theory. We mainly focus on low-energy threshold states far below the pion mass, i.e., the theory is pionless, with explicit pion degrees of freedom and their interaction effectively integrated out. We note that the breakdown scale of the pionless EFT (i.e., $\left.{ }^{\star} \Lambda \sim m_{\pi}\right)$ is different from that in Sec. III. To establish the flavor extrapolation of the meson-neutron scattering lengths we have explicitly included pions in the SU(4) model with vector meson exchange. Once this is settled, we concentrate on the states near the threshold by utilizing the pionless theory.

A power counting rule [62 64 for two-body contact interactions for such finely-tuned systems, incorporated with the power divergence subtraction scheme, is most suitable for renormalizing the strongly interacting twobody sector. In this section, we display the effective Lagrangian of the three-body system, where we denote the generic flavored meson field as $K$ for brevity, but what it basically stands for is the flavor extrapolated meson field $K(x)$, implicitly dependent on the parameter $0 \leq x \leq 1$. Note here that the actual meson charge is irrelevant in our context without Coulomb interaction, so that the change in the charge from the antikaon $K^{-}$(for $x=0$ ) to the $D^{0}$ meson (for $x=1$ ) should not be a matter of concern.

The non-relativistic effective Lagrangian for the threebody system, consistent with the usual low-energy symmetries, like parity invariance, charge conjugation, timereversal invariance, and small velocity Lorentz invariance, can be constructed as

$$
\mathcal{L}=\mathcal{L}_{K}+\mathcal{L}_{n}+\mathcal{L}_{\text {2-body }}+\mathcal{L}_{3 \text {-body }}
$$

As regards the fundamental fields, the neutron is represented by a two-component (spin-doublet) spinor $\psi_{n}$, and the flavor extrapolated meson is represented by a spin-singlet field, $K(x)$. The standard forms of the single "heavy" particle Lagrangians, $\mathcal{L}_{K}$ and $\mathcal{L}_{n}$, at the leading order are well known [31, 39, 65, 66], and their expressions will not be repeated here. However, in the two-body sector, we explicitly spell out the relevant interaction parts of the leading order Lagrangian $\mathcal{L}_{2 \text {-body }}$, projecting the dominant $s$-wave terms. When the $n n$ and flavor extrapolated $n K$ scattering lengths are much larger than the typical length scales of short-range interactions, the two-body interactions can be expressed by means of contact terms with couplings $g_{n K}$ and $g_{n n}$, parametrizing the short-distance UV physics:

$$
\begin{aligned}
\mathcal{L}_{2 \text {-body }}^{\text {int }}= & -g_{n n}\left(\psi_{n}^{T} P_{(n n)}^{\left({ }^{1} \mathrm{~S}_{0}\right)} \psi_{n}\right)^{\dagger}\left(\psi_{n}^{T} P_{(n n)}^{\left({ }^{1} \mathrm{~S}_{0}\right)} \psi_{n}\right) \\
& -g_{n K} \phi_{K}^{\dagger} \psi_{n}^{\dagger} \psi_{n} \phi_{K}+\cdots,
\end{aligned}
$$

where the spin-singlet projection operator is defined as

$$
P_{(n n)}^{\left({ }^{1} \mathrm{~S}_{0}\right)}=-\frac{i}{2} \sigma_{2} .
$$

In this context, it is convenient to describe the threebody system in terms of auxiliary diatom or dihadron fields [31, 65, 66], namely, a spin-singlet nn-dibaryon field $s_{(n n)}$, and a spin-doublet $n K$-dihadron field $d_{(n K)}$. Through a Gaussian integration followed by a redefinition of the fields, the contact terms in the above two-body Lagrangian can be re-expressed as

$$
\begin{aligned}
\mathcal{L}_{d(n K)}= & \frac{1}{g_{n K}} d_{(n K)}^{\dagger} d_{(n K)} \\
& -\left[d_{(n K)}^{\dagger} \psi_{n} \phi_{K}+\text { h.c. }\right]+\cdots \\
\mathcal{L}_{s(n n)}= & \frac{1}{g_{n n}} s_{(n n)}^{\dagger} s_{(n n)} \\
& -\left[s_{(n n)}^{\dagger}\left(\psi_{n}^{T} P_{(n n)}^{\left({ }^{1} \mathrm{~S}_{0}\right)} \psi_{n}\right)+\text { h.c. }\right]+\cdots,
\end{aligned}
$$

where the ellipses in the above expressions denote the sub-leading interaction terms irrelevant in the context of our analysis.

Our three-body system exhibits the Efimov effect in the ZCL, as a straightforward consequence of the unitarity of the two-body amplitude, which is also demonstrated by the numerical analysis below. Following the basic EFT tenet, this necessitates the additional inclusion of a leading order non-derivatively coupled threebody contact term in the Lagrangian for renormalization (see, e.g., Refs. [31, 65 67] for details). In our case, such a three-body term is given by

$$
\begin{aligned}
\mathcal{L}_{3 \text {-body }}= & \mathcal{L}_{n d(n K)}^{\left({ }^{1} \mathrm{~S}_{0}\right)}+\cdots \\
\mathcal{L}_{n d(n K)}^{\left({ }^{1} \mathrm{~S}_{0}\right)}=-\frac{m_{K} g_{s}(\mu)}{\mu^{2}} & \left(d_{(n K)}^{T} P_{(n d)}^{\left({ }^{1} \mathrm{~S}_{0}\right)} \psi_{n}\right)^{\dagger} \\
& \times\left(d_{(n K)}^{T} P_{(n d)}^{\left({ }^{1} \mathrm{~S}_{0}\right)} \psi_{n}\right)+\cdots
\end{aligned}
$$

with the spin-singlet projection operator

$$
P_{(n d)}^{\left({ }^{1} \mathrm{~S}_{0}\right)}=-\frac{i}{\sqrt{2}} \sigma_{2},
$$

and $g_{s}(\mu)$, an a priori unknown scale dependent threebody coupling. Here we especially choose to promote to the leading order, only the three-body interaction corresponding to the spin-singlet elastic channel, $n d_{(n K)} \rightarrow$ $n d_{(n K)}$. The ellipses denote other allowed three-body interaction terms to be treated generally as sub-leading. It is important to note that precisely the presence of the asymptotic limit cycle in our case gives us the freedom to choose any one of the channels to promote the corresponding three-body term. 


\section{B. Three-body integral equation}

We now consider the integral equations for the mesonneutron-neutron three-body system in the total spinsinglet channel. Such a system of equations can be constructed either by combining the dibaryon field $s_{(n n)}$ and the flavored meson field $K$ or by combining the dihadron field $d_{(n K)}$ and the neutron field $n$. The system of equations consists of two kinds of Faddeev-like partitions: a direct (elastic) hadron exchange channel $n d_{(n K)} \rightarrow n d_{(n K)}$ (denoted as $\left.t_{a}\right)$, and a hadron rearrangement (inelastic) channel $n d_{(n K)} \rightarrow s_{(n n)} K$ (denoted as $\left.t_{b}\right)$. For concreteness, the coupled-channel threebody equations for the half-off-shell amplitudes $t_{a}$ and $t_{b}$ are diagrammatically displayed (omitting the three-body contact terms for brevity) in Fig. 7 [39, 68 70].

It is straightforward to obtain these equations following the Feynman rules from the Lagrangian presented in the previous subsection. After $s$-wave projections, we obtain the following expressions:

$$
\begin{aligned}
t_{a}\left(p^{\prime}, p ; E\right)= & m_{K}\left\{\frac{1}{2 p^{\prime} p} \ln \left[\frac{p^{2}+p^{2}+a p^{\prime} p-2 \mu_{(n K)} E}{p^{2}+p^{2}-a p^{\prime} p-2 \mu_{(n K)} E}\right]-\frac{g_{s}(\Lambda)}{\Lambda^{2}}\right\} \\
& -\frac{m_{K}}{\pi \mu_{(n K)}} \int_{0}^{\Lambda} d l l^{2}\left\{\frac{1}{2 p^{\prime} l} \ln \left[\frac{p^{\prime 2}+l^{2}+a p^{\prime} l-2 \mu_{(n K)} E}{p^{\prime 2}+l^{2}-a p^{\prime} l-2 \mu_{(n K)} E}\right]-\frac{g_{s}(\Lambda)}{\Lambda^{2}}\right\} \frac{t_{a}(l, p ; E)}{\frac{1}{a_{d(n K)}}-\sqrt{-2 \mu_{n K} E+\frac{\mu_{(n K)} \mu_{n(n K)}}{\mu^{2}-i 0^{+}}}} \\
& -\frac{\sqrt{2}}{\pi} \int_{0}^{\Lambda} d l \frac{l}{p^{\prime}} \ln \left[\frac{p^{\prime 2}+b l^{2}+p^{\prime} l-M_{n} E}{p^{\prime 2}+b l^{2}-p^{\prime} l-M_{n} E}\right] \frac{t_{b}(l, p ; E)}{\frac{1}{a_{s(n n)}}-\sqrt{-M_{n} E+\frac{M_{n}}{2 \mu_{K(n n)}} l^{2}-i 0^{+}}}
\end{aligned}
$$

and

$$
\begin{aligned}
t_{b}\left(p^{\prime}, p ; E\right)= & \frac{M_{n}}{\sqrt{2} p^{\prime} p} \ln \left[\frac{b p^{2}+p^{2}+p^{\prime} p-M_{n} E}{b p^{\prime 2}+p^{2}-p^{\prime} p-M_{n} E}\right] \\
& -\frac{M_{n}}{\sqrt{2} \pi \mu_{(n K)}} \int_{0}^{\Lambda} d l \frac{l}{p^{\prime}} \ln \left[\frac{b p^{\prime 2}+l^{2}+p^{\prime} l-M_{n} E}{b p^{\prime 2}+l^{2}-p^{\prime} l-M_{n} E}\right] \frac{t_{a}(l, p ; E)}{\frac{1}{a_{d(n K)}}-\sqrt{-2 \mu_{(n K)} E+\frac{\mu_{(n K)}}{\mu_{n(n K)}} l^{2}-i 0^{+}}} .
\end{aligned}
$$

Here $E$ is the total center-of-mass kinetic energy of the three-body system, $p$ and $p^{\prime}$ are the initial and final state momenta, and $\mu_{(n K)}(x)=M_{n} m_{K}(x) /\left[M_{n}+m_{K}(x)\right]$ is the meson-neutron reduced mass. The $s$-wave meson-neutron and neutron-neutron scattering lengths, $a_{d(n K)} \equiv a_{d(n K)}(x)$ and $a_{s(n n)}$, respectively, are given as

$$
\begin{gathered}
\frac{1}{a_{d(n K)}(x)}=\frac{2 \pi}{\mu_{(n K)}(x) g_{n K}}+\mu_{p . d . s .} \\
\frac{1}{a_{s(n n)}}=\frac{4 \pi}{M_{n} g_{n n}}+\mu_{p . d . s .},
\end{gathered}
$$

when the power divergence subtraction scheme 6264] is adopted with subtraction scale $\mu_{\text {p.d.s. } \sim} \sim$ $m_{\pi}$. Furthermore, we introduce the neutron-dihadron $(n+n K)$-reduced mass as $\mu_{n(n K)}(x)=M_{n}\left[M_{n}+\right.$ $\left.m_{K}(x)\right] /\left[2 M_{n}+m_{K}(x)\right]$, along with the two other mass dependent parameters:

$$
a(x)=\frac{2 \mu_{(n K)}(x)}{m_{K}(x)}, \quad b(x)=\frac{M_{n}}{2 \mu_{(n K)}(x)} .
$$

In the above expressions, we re-emphasized the dependence on the extrapolation parameter $x$ at a general unphysical point $x \neq 0,1$. The coupled integral equations must be numerically solved to obtain the nonperturbative solutions to the three-body problem. It is important to note that these integral equations must be regularized, e.g., by introducing the sharp momentum cut-off $\Lambda$ to remove ambiguities in the asymptotic phase with the cut-off taken to infinity 67, 71]. The resulting cut-off scale dependence is renormalized by introducing the leading order three-body contact interaction term displayed in Eq. (28) with a cut-off dependent running coupling, $g_{s}(\mu=\Lambda) \propto \ln (\Lambda)$, leading to a RG limit cycle.

\section{Asymptotic analysis}

In order to determine whether the three-body system exhibits the Efimov effect, it is instructive to examine the asymptotic behavior (both in the scaling as well as unitary limits) of the integral equations with the cutoff removed (i.e., $\Lambda \rightarrow \infty$ ) and the three-body contact interaction excluded (see Refs. [31, 36, 66] for details). In this limit, the scale of the off-shell momenta can be considered very large in comparison with the inverse two-body scattering lengths and the eigenenergies, 


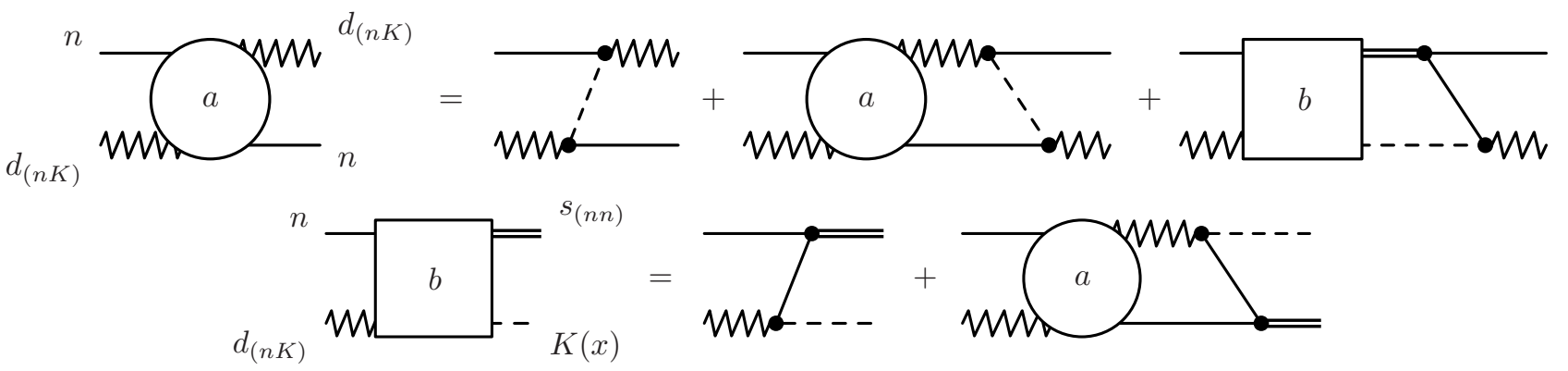

FIG. 7. Feynman diagrams for the three-body coupled integral equations where the three-body contact terms are omitted for brevity. The solid and dashed lines represent the bare propagators of $n$ and $K(x)$, respectively, and the double and zigzag lines stand for the dressed dihadron propagators of $s_{(n n)}$ and $d_{(n K)}$, respectively.

$E=-B_{d}<0$ of the three-body bound stat7, i.e., $p^{\prime}, l \gg 1 / a_{d(n K)}, 1 / a_{s(n n)}, \sqrt{2 \mu_{(n K)} B_{d}}, \sqrt{M_{n} B_{d}}$. In particular, the asymptotic solution to the amplitudes is expected to follow a power law: $t_{a, b}\left(p^{\prime}\right) \propto p^{\prime s-1}$. It is then straightforward to show that the coupled integral equations in this regime get reduced into a single transcendental equation which may be solved for the exponent $s$. By using the meson masses in the physical limits, i.e., for the antikaon $K^{-}(x=0)$ and the charmed meson $D^{0}(x=1)$, as given in Table प we obtain in each case a pair of imaginary solutions, $s= \pm i s_{0}^{\infty}$, with the respective transcendental number $s_{0}^{\infty}$ given by

$$
s_{0}^{\infty}= \begin{cases}1.03069 & \text { for } K^{-} n n \\ 1.02387 & \text { for } D^{0} n n\end{cases}
$$

This confirms the previously obtained result by Braaten and Hammer (see Fig. 52 of Ref. 31)). In fact, the imaginary solutions of $s$ in the unphysical domain, i.e., $0<x<1$, are continuously connected in the process of interpolating the flavored meson mass $m \equiv m_{K}(x)$ in between the physical limits $m_{K^{-}}(x=0)$ and $m_{D^{0}}(x=1)$, as depicted in Fig. 8 . We thereby conclude that the coupled integral equations are ill-defined in the asymptotic limit which means that formally Efimov effect must be manifest in the three-body system, not only at the physical limits $(x=0,1)$, but at all intermediate unphysical points. Of course one should bear in mind the formal nature of the above results which follow from a leading order asymptotic analysis corresponding to the scaling limit of all the two-body interactions. But the more robust physical realization of the Efimov spectrum, i.e., whether or not it lies within an Efimov window, evidently relies on the nature of possible crucial range effects.

7 Here we use the notation, $B_{d}=-E>0$, to denote the absolute value of the trimer binding energy, measured with respect to three-particle break-up threshold. This is to distinguish $B_{d}$ from our notation for the relative trimer energy, $B_{T}=B_{d}-E_{D}>0$, measured from the particle-dimer break-up threshold energy of $E_{D}$ [also, see Eq. 40]].

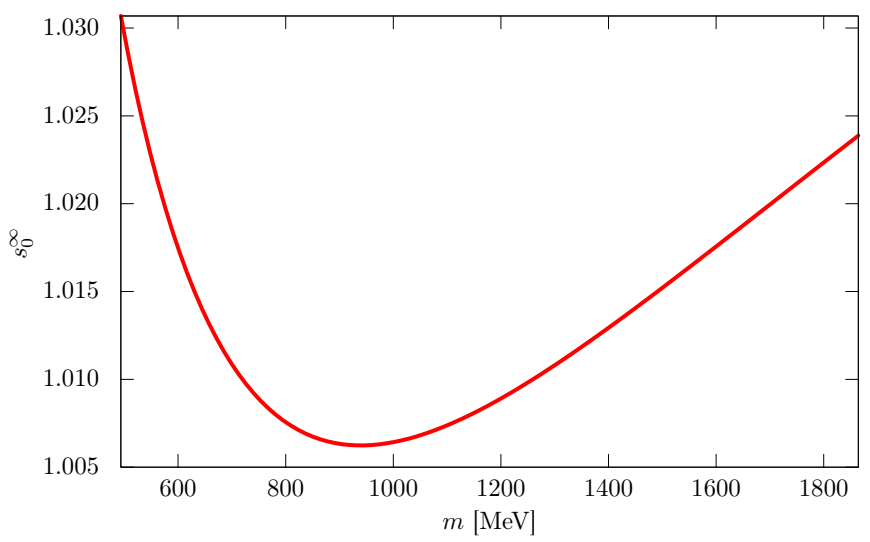

FIG. 8. Flavored meson mass dependence on the asymptotic parameter $s_{0}^{\infty}$ for $m_{K^{-}}<m<m_{D^{0}}$.

\section{Numerical results}

As already mentioned, the numerical evaluations of the three-body integral equation require the meson-neutron and neutron-neutron $s$-wave scattering lengths as the principal two-body input to our leading order EFT analysis. First, our numerical results are displayed with the spin-singlet $s$-wave $n n$ scattering length taken as its physical value, $a_{s(n n)}=-18.63 \mathrm{fm}$ [72]. Second, we try to demonstrate the remnant universal features of the nonasymptotic results in the physical sectors whereby we neglect the absorptive contributions of the decay/coupled channels for simplicity. For the purpose of extrapolating to the unphysical domain to probe the unitary limit, it is convenient to use our predicted scattering lengths in the ZCL, as displayed in Eq. (20) in the physical limits $(x=0,1)$, namely,

$$
\begin{aligned}
a_{d\left(n K^{-}\right)} & \equiv a_{d(n K)}(x=0)=a_{0, K^{-} n}^{\mathrm{ZCL}}=-0.394 \mathrm{fm}, \\
a_{d\left(n D^{0}\right)} & \equiv a_{d(n K)}(x=1)=a_{0, D^{0} n}^{\mathrm{ZCL}}=4.141 \mathrm{fm} .
\end{aligned}
$$

We thereby solve the non-asymptotic integral equations (30) and (31) to obtain the three-body energy eigenvalues as a function of the sharp cut-off regulator $\Lambda$. 
We first include the three-body force containing the coupling $g_{s}(\Lambda)$ to investigate into its approximate limit cycle nature at non-asymptotic momenta/energies. Later, we shall focus on the results excluding the threebody term to investigate into the behavior of the threebody eigenenergies of the physical sectors (i.e., $x=0,1$ limits), generated exclusively from the two-body dynamics. In particular, the role of the cut-off $(\Lambda)$ dependence of the results is investigated which can crucially determine whether the three-body bound state formation is formally supported in the low-energy EFT with zerorange approximation. Furthermore, in the context of the ZCL approach, a study of the first "critical" cut-off $\Lambda_{c}^{(n=0)}$ (defined later in the sub-section) excluding the three-body force is presented, whose variation with the extrapolation parameter $x$ reveals vital information on three-body universality. Finally, in association with our findings in the two-body sector, the above results may be used to predict the likelihood of realistic Efimov-like bound states. A brief description of our methodology in the numerical solution to the integral equations is presented in Appendix A.

We then present our result for the RG limit cycle for the three-body contact interaction coupling $g_{s}(\Lambda)$ in the $K^{-} n n\left(D^{0} n n\right)$ system. For a proper estimation of this coupling a prior knowledge of a three-body observable, such as the $s$-wave three-particle (particle-dimer) scattering length or the trimer binding energy is required, neither of which is currently available. However, even in the absence of such a three-body datum, we may study the RG behavior of this coupling by fixing any presumably small/near-threshold value of the trimer binding energy, say, $B_{d}, B_{T}=0.1 \mathrm{MeV}$, and varying the cut-off scale $\Lambda$. Various qualitative features concerning three-body universality can be deduced thereof.

Figure 9 demonstrates the typical cyclic or quasi-logperiodic behavior of the coupling $g_{s}(\Lambda)$ for the $K^{-} n n$ and $D^{0} n n$ systems, reflecting an approximate RG limit cycle in each case. The periodic divergences of the coupling are associated with the sequence of successive formation of three-body bound states. For the $D^{0} n n$ system the first three bound states are evident for $\Lambda<10^{5} \mathrm{MeV}$, and for the $K^{-} n n$ system only the first two are visible in this range. The qualitative feature of the limit cycle plots remains unchanged whether the binding energy, $B_{d}$ $\left(B_{T}\right)$, is chosen approaching the three-particle (particledimer) break-up threshold, as shown in the figure, or chosen somewhat away from the threshold; there is basically a slight downward and rightward shift of each curve with increasing $B_{d}\left(B_{T}\right)$ because the interaction becomes more and more attractive.

For the running coupling $g_{s}(\Lambda)$, the characteristic periodicity, with $\Lambda=\Lambda^{(N)} \forall N \in \mathbb{N}$, can be expressed as

$$
g_{s}\left(\Lambda^{(1)}\right)=g_{s}\left(\Lambda^{(N+1)}\right) \text { with } \Lambda^{(N+1)}=\Lambda^{(1)} \exp \left(\frac{N \pi}{s_{0}}\right) \text {, }
$$

where $s_{0}$ is a real three-body parameter reminiscent of

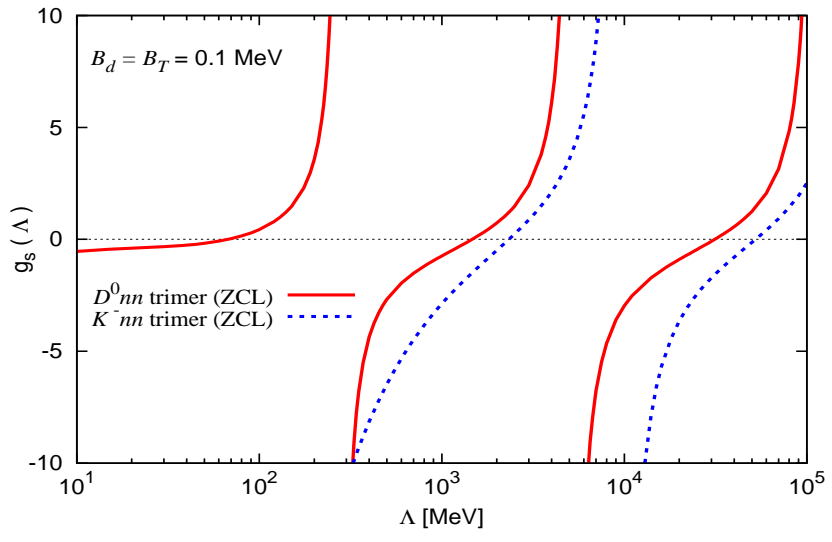

FIG. 9. RG Limit cycle for the three-body coupling $g_{s}$ as a function of the sharp momentum cut-off $\Lambda$. The pertinent integral equations are solved with the input two-body scattering lengths from Eq. (36) in the zero coupling limit for fixed trimer eigenenergies, namely, $B_{d}=0.1 \mathrm{MeV}$ for $K^{-} n n$, measured with respect to the three-particle break-up threshold, and $B_{T}=B_{d}-E_{D}=0.1 \mathrm{MeV}$ for $D^{0} n n$, measured with respect to the particle-dimer break-up threshold energy of $E_{D}=1.82 \mathrm{MeV}$.

the corresponding asymptotic limit cycle value of $s_{0}^{\infty}=$ $1.03069\left(s_{0}^{\infty}=1.02387\right)$ that we found earlier for the $K^{-} n n\left(D^{0} n n\right)$ system. Physically, $s_{0}$ gives a measure of the residual approximate discrete scale invariance surviving in the non-asymptotic regime. In particular, the running coupling vanishes quasi-periodically at a discrete set of cut-off values, expressed as $\Lambda_{0}^{(N+1)}=\Lambda_{0}^{(1)} \exp \left(\frac{N \pi}{s_{0}}\right)$. It is to be noted that $s_{0}^{\infty}$ is a universal number, depending only on the gross features of the three-particle system, like ratios of the respective masses of the three particles involved, or the overall spin and isospin quantum numbers of the system. On the other hand, the non-universal number $s_{0}$ deviates from $s_{0}^{\infty}$ primarily due to cut-off dependent effects, implying an implicit dependence on $N$ itself, i.e., $s_{0} \equiv s_{0}^{(N)}$. Additional non-asymptotic parametric dependence on the trimer binding energy, three-body coupling and the two-body scattering lengths, can further influence the numerical value of $s_{0}^{(N)}$. In other words, $s_{0}^{(N)}$ being the outcome of a numerical RG is purely numerical by nature, and thereby difficult to ascribe a unique definition in terms of a analytical expression. However, reasonably good estimates are obtained by taking the successive discrete cut-offs, say, $\Lambda_{0}^{(N)}$ and $\Lambda_{0}^{(N+1)}$ for vanishing coupling $g_{s}\left(\Lambda_{0}^{(N)}\right)=g_{s}\left(\Lambda_{0}^{(N+1)}\right)=0$, and using the closed-form definition:

$$
s_{0}^{(N)}=\frac{\pi}{\ln \left(\frac{\Lambda_{0}^{(N+1)}}{\Lambda_{0}^{(N)}}\right)} .
$$

A crucial observation in this definition is that the sequence of the non-asymptotic numbers $s_{0}^{(N)}$, as obtained above, gradually converges to the asymptotic limit value 


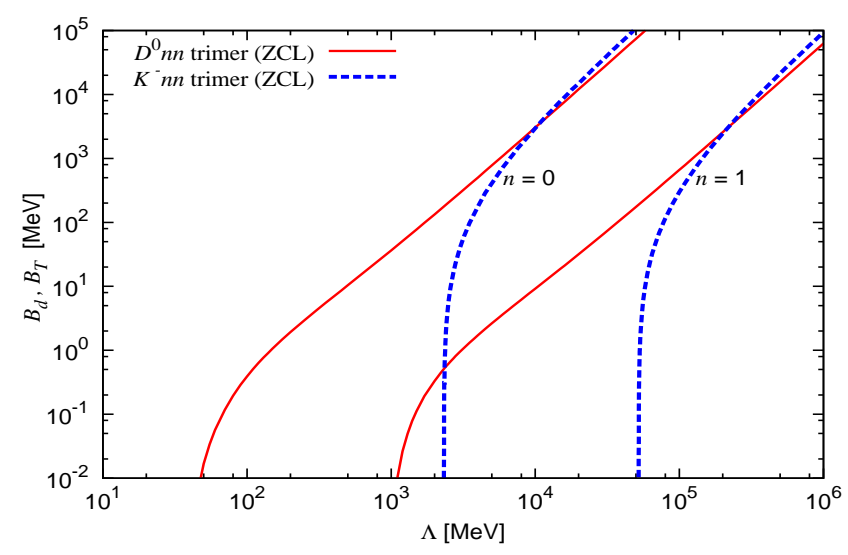

FIG. 10. Binding energies for the ground $(n=0)$ and first $(n=1)$ excited level states as a function of the sharp momentum cut-off $\Lambda$, excluding the three-body force. The pertinent integral equations are solved with the input two-body scattering lengths from Eq. (36) in the zero coupling limit. For the $K^{-} n n$ system, the trimer binding energy $B_{d}$, is measured with respect to the three-particle break-up threshold, while for the $D^{0} n n$ system the binding energy, $B_{T}=B_{d}-E_{D}$, is measured with respect to the particle-dimer break-up threshold energy, $E_{D}=1.82 \mathrm{MeV}$.

$s_{0}^{\infty}$ beyond a suitably large $N>0$. Mathematically, stated: for $\epsilon>0, \exists N_{0} \in \mathbb{N}$, such that

$$
\left|s_{0}^{(N)}-s_{0}^{\infty}\right|<\epsilon, \quad \forall N>N_{0} .
$$

An alternative method of extraction of the parameter $s_{0}$ is presented in Appendix B.

In the following numerical study, we shall exclude the three-body force since there is no three-body input datum available to fix the coupling $g_{s}$. In Fig. 10, the results for the trimer energy of the physical systems, $K^{-} n n(x=0)$ and $D^{0} n n(x=1)$, are summarized. In the figure, the ground $(n=0)$ and the first excited $(n=1)$ state binding energies, $-E=B_{d}>0$, of the $K^{-} n n$ Borromean trimer [32] (with both $a_{d\left(n K^{-}\right)}, a_{s(n n)}<0$ ), measured with respect to the three-particle breakup threshold, are plotted as a function of $\Lambda$. The same figure also displays the corresponding eigenenergies, $B_{T}=B_{d}-E_{D}$, of the $D^{0} \mathrm{nn}$ trimer state, measured with respect to the particle-dimer $\left(n+D^{0} n\right)$ break-up threshold energy $E_{D}$. Note that the threshold value of the $D^{0} n$ dimer binding energy, which is given by

$$
E_{D}=\frac{1}{2 \mu_{(n D)} a_{d(n D)}^{2}},
$$

is obtained as $E=-E_{D}=-1.82 \mathrm{MeV}$, using the $D^{0} n$ reduced mass $\mu_{(n D)}=\frac{M_{n} m_{D}}{M_{N}+m_{D}}$, and the corresponding ZCL scattering length given in Eq. (36). It is to be noted that the value $-E_{D}$ closely matches with the value of the $D^{0} n$ bound state scattering amplitude pole position, $E_{h}=-1.85 \mathrm{MeV}$ (see Fig. 2).
Figure 10 clearly indicates the increasing magnitude of the binding energies as the cut-off scale $\Lambda$ increases. In this figure, the first branch refers to the ground state of the $D^{0} n n\left(K^{-} n n\right)$ system, which appears as a shallow threshold state at the so-called "critical" cut-off value of $\Lambda_{c}^{(\mathrm{n}=0)} \simeq 38.4 \mathrm{MeV}(\simeq 2.3 \mathrm{GeV})$. The state then becomes increasingly deeply bound with increasing cut-off, while a second branch (first excited state) appears at the critical value, $\Lambda_{c}^{(\mathrm{n}=1)} \simeq 1 \mathrm{GeV}(\simeq 52.2 \mathrm{GeV})$, which in its turn gets progressively deeper. Continuing in this manner an infinite tower of excited states emerges from the zero energy threshold as $\Lambda \rightarrow \infty 8$ Again, as pointed out earlier in the context of Fig. 9, only the ground state $D^{0} n n$ trimer is likely to satisfy conditions that may formally qualify it as an Efimov-like state.

Let us finally study the flavor extrapolation from the $K^{-} n n$ system $(x=0)$ to the $D^{0} n n$ system $(x=1)$. As seen in Sec. IIC a continuous extrapolation of the meson-neutron system from $K^{-} n$ to $D^{0} n$ (see Fig. 3) in ZCL yielded an unitary limit of the meson-neutron interaction in the proximity of $x \sim 0.6$. Here excluding the three-body force we investigate the behavior of the "critical point" $\Lambda_{c}^{(0)}$, corresponding to the ground state $(n=0)$, as a function of the extrapolation parameter $x$ for fixed (absolute or relative) trimer binding energy $\left(B_{d}\right.$ or $B_{T}$ ). In other words, we perform an extrapolation in the interval $x \in[0,0.615)$, i.e., along the three-particle break-up threshold with a fixed value of $B_{d}$, and continue further in the interval $x \in(0.615,1]$, i.e., along the particle-dimer break-up threshold with a fixed value of $B_{T}=B_{d}-E_{D}$, where $E_{D} \equiv E_{D}(x)$ in the latter interval is itself $x$ dependent. It may be noted that level energies chosen too close to the thresholds can lead to numerical instabilities in the vicinity of the unitary limit. We have, therefore, preferred non-zero but near threshold binding energies, say, $B_{d}, B_{T} \gtrsim 0.001 \mathrm{MeV}$ to suit our purpose of demonstration.

Figure 11 displays our results where the meson mass and the $s$-wave meson-neutron scattering length are simultaneously extrapolated from $x=0$ to $x=1$. The fact that there is a large change ( $\sim 3$ orders of magnitude) in the critical cut-off along $x \in[0,0.615)$ compared to a rather nominal change along $x \in(0.615,1]$, suggests that the $D^{0} \mathrm{nn}$ system is much more likely to be found in the domain of three-body universality and yields an Efimov-like bound state in comparison to the $K^{-} n n$ system. This reflects the much larger scattering length, $a_{0, D^{0} n}^{\mathrm{ZCL}} \sim 4 \mathrm{fm}$, than that in the strangeness sector, $a_{0, K^{-}{ }_{n}}^{\mathrm{ZCL}} \sim-0.4 \mathrm{fm}$. The horizontal dotted line in the above figure indicates the one-pion threshold, the upper

\footnotetext{
8 Because here we exclude the three-body force, the critical point associated with the ground state, denoted here as $\Lambda_{c}^{(n=0)}$, corresponds to the first zero at $\Lambda=\Lambda_{0}^{(N=1)}$ of the three-body contact interaction, i.e., $g_{s}\left(\Lambda_{0}^{(N=1)}\right)=0$ in Fig. 9 for fixed trimer binding energy.
} 


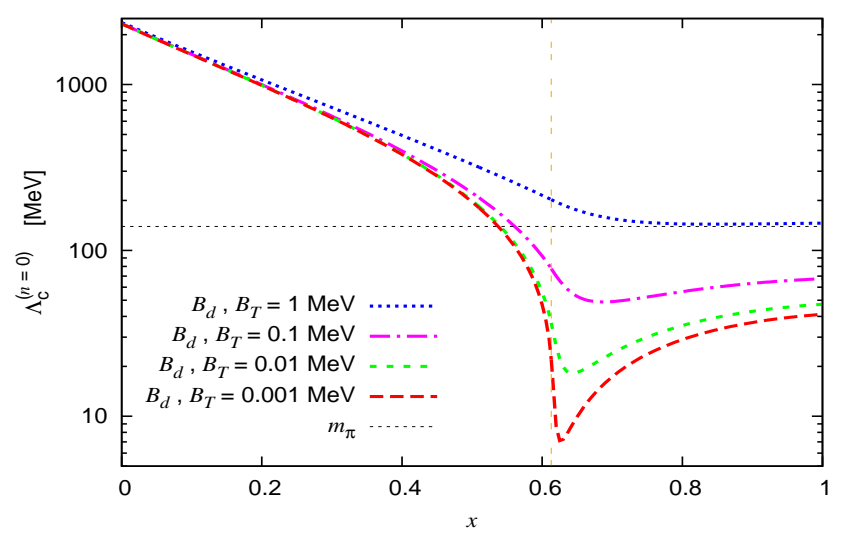

FIG. 11. Extrapolation of the critical cut-off $\Lambda_{c}^{(0)}$, associated with the ground state $(n=0)$ for fixed binding energies $\left(B_{d}\right.$ or $B_{T}$ ) of the meson-neutron-neutron bound state, measured along the three-particle or particle-dimer break-up threshold. The double dashed vertical line at $x=0.615$ denotes the unitary limit. The plot corresponds to the zero coupling limit with input scattering lengths, as given in Eq. (36). The threebody force is excluded in these results.

cut-off of the pionless EFT employed in this analysis $9 \mathrm{~A}$ direct comparison with the behavior of the critical cut-off indicates that a $D^{0} n n$ ground state trimer is clearly supported in the realm of the low-energy EFT framework, lying well below the hard scale ${ }^{\star} \Lambda \sim m_{\pi}$, while the same for the $K^{-} n n$ system lies far above the applicability of the low-energy EFT, and thus, can be considered effectively unbound by Efimov attraction.

\section{DISCUSSION AND SUMMARY}

In this work, we have explored the two and three-body universal physics associated with a three-particle cluster state of two neutrons and a flavored meson, i.e., an antikaon $K^{-}$or a $D^{0}$ meson. We have demonstrated that the meson-neutron scattering length can become infinitely large in an idealized limit, around which universality governs the physics of two-body and three-body systems. This offers an interesting prospect that a possible remnant of this universal physics may be observed in the physical hadronic systems.

First, we have studied the two-body meson-neutron systems. Motivated by the experimental evidences that the $K^{-} n\left(D^{0} n\right)$ system has no quasi-bound state (one quasi-bound state), we construct a theoretical coupledchannel model to describe both $K^{-} n$ and $D^{0} n$ systems with a single flavor extrapolation parameter $x$. This

9 In fact, it may be noted that the cut-off scale of the shortdistance two-body interaction should be taken still larger since the one-pion exchange process is forbidden in the $K^{-} n$ or $D^{0} n$ system. model enables the extrapolation to unphysical quark masses between the strange $(x=0)$ and the charm $(x=1)$ flavors limits, providing a natural mechanism to tune the strength of the meson-neutron interaction. While the universal features are not very prominent due to the coupled-channel effects, it was shown that the meson-neutron interaction can reach resonant conditions with large two-body scattering length around $x \sim 0.6$ in the zero coupling limit (ZCL) with the channel couplings switched off. We further investigated two-body universality by comparing the eigenmomentum of the system with that predicted solely by the two-body scattering lengths. This led to the identification of the entire region $x \gtrsim 0.3$ as the two-body universal window in the ZCL, including the charm sector $(x=1)$. Moreover, the universal prediction reasonably works in the full coupledchannel model with complex meson-neutron scattering lengths in the extrapolation region above $x \gtrsim 0.8$. This indicates that universality governs not only the idealized ZCL scenario but also the physical $D^{0} n$ system, both being continuously connected. One can then conclude that the physical charm sector is likely to follow the predictions of universality, even with the couplings to the decay channels included.

Next, we investigated three-body universality by employing a low-energy cluster EFT. In a simplified approach, we especially neglected the absorptive influence of sub-threshold decay channels which amounts to considering the $s$-wave meson-neutron scattering lengths to be real-valued. A leading order EFT analysis allowed us to investigate the meson-neutron-neutron system in the scaling limit (zero-range approximation) where the universal physics was determined only by the two $s$-wave scattering lengths $a_{d(n K)}$ and $a_{s(n n)}$. In this work $a_{s(n n)}$ was kept fixed to the physical value, while $a_{d(n K)} \equiv$ $a_{d(n K)}(x)$ was varied as a function of $x$. The introduction of a sharp momentum cut-off $\Lambda$ in the integral equations led to the breaking of continuous scale invariance resulting in the introduction of range-like effects. In this context, it may be noted that effective range corrections were not explicitly considered in this qualitative leading order analysis. We anticipate that the range correction would not be significant in the charm sector based on the two-body analysis in Figs. 5 and 6. For definite conclusion, however, it is important to explicitly examine the range correction, which is left as a future prospect of this work.

An immediate consequence of the breaking of continuous scale invariance in the three-body sector is the appearance of a discrete scaling symmetry in the solutions to the integral equations that can be associated with an asymptotic RG limit cycle. In this context, Fig. 8 suggests that the asymptotic limit cycle between the $K^{-} n n$ and $D^{0} n n$ systems continuously exists for all intermediate values of $x$ so that the respective asymptotic parameters $s_{0}^{\infty}=1.03069$ (strange sector) and $s_{0}^{\infty}=1.02387$ (charm sector) are smoothly connected. This leads us to conclude that by neglecting the influence of decay chan- 
nels and range corrections, the Efimov effect can always be manifested in the solutions to the three-body coupled integral equations. In fact, from Fig. 9, we confirmed approximate RG limit cycle behavior (with quasilog-periodicity) of the three-body running coupling $g_{s}(\Lambda)$ in the non-asymptotic solutions for each system.

Next, the binding energies of the three-body systems in the physical limits were obtained as a function of the cut-off $\Lambda$. For the sake of simplicity we excluded the dependence on the three-body force with the unknown coupling $g_{s}$. We found that the binding energies increase with increasing $\Lambda$, and the various level states emerge in order from the zero energy threshold, starting from a deepest (ground) state that appears at a certain critical value, $\Lambda=\Lambda_{c}^{(0)}$. It may be noted that a larger value of the momentum cut-off leads to additional inclusion of ultraviolet physics into the theory arising from high energy modes. This evidently means greater attraction in the system leading to larger three-body binding energies and the emergence of new level states. It clearly emerges from our results that the Efimov spectrum is much steeper on the negative side of the scattering lengths. With the much smaller critical cut-offs for the $D^{0} n n$ level states than those of the $K^{-} n n$ system, it is straightforward to conclude that the $D^{0} n n$ trimer states are manifested more easily.

Finally, as shown in Fig. 11, the behavior of the lowest critical cut-off $\Lambda_{c}^{(0)}$ associated with the emergence of near threshold (three-body) ground states was obtained as a function of $x$ using the extrapolated meson-neutron scattering length in the ZCL. Clearly with smaller and smaller chosen values of $B_{d}$ or $B_{T}, \Lambda_{c}^{(0)}$ converged to the point $\left(x \simeq 0.6, \Lambda_{c}^{(0)} \simeq 0\right)$, at which near threshold states were realized corresponding to the vanishing scale of twobody unitarity. As $x$ is taken away from unitarity toward the physical limits, $x=0,1, \Lambda_{c}^{(0)}$ becomes larger, indicating larger two-body interaction strengths needed to form three-body bound states. Clearly, the $x=1$ physical limit $\left(D^{0} n n\right.$ system) is located much closer to the unitary limit at $x \simeq 0.6$ than the $x=0$ physical limit $\left(K^{-} n n\right.$ system). This naturally indicates that a $D^{0} n n$ ground state trimer can be realized in the ZCL in pionless EFT framework with $\Lambda_{c}^{(0)}$ lying well below the pion mass. In fact, this is a straightforward consequence of the large $D^{0} n$ scattering length in the ZCL, i.e., $a_{0, D^{0} n}^{\mathrm{ZCL}} \sim 4$ fm, which traces back to the existence of $\Sigma_{c}(2800)$ near the $D^{0} n$ threshold. While for the $K^{-} n n$ system, with $\Lambda_{c}^{(0)} \gtrsim 2 \mathrm{GeV}$, no physically realizable mechanism in the context of a low-energy EFT can generate sufficient interaction strength to form bound states. In a sense, the much steeper Efimov spectrum for the $K^{-} n n$ system is consistent with the fact that such Borromean Efimov trimers are by nature extremely difficult to form.

In retrospect, through a simplistic qualitative analysis we have presented here a rather idealistic scenario whereby universal Efimov physics may be realized in a meson-neutron-neutron system, despite the smallness of the meson-neutron scattering lengths. Such a scenario may very well be unrealistic given the rather delicate nature of the Efimov-like physics which may easily become obscured or wiped out altogether due to range and coupled-channel effects with $C_{i 1}=C_{1 i} \neq 0(i=2,3,4)$ restored. Nevertheless, it may still be somewhat interesting to pursue further studies to assess the exact nature of the $D^{0} n n$ system which has never been previously considered as a possible candidate for a hadronic molecule. Having said that, it must also be borne in mind that the dynamics of the sub-threshold decay channels which were systematically neglected in this work can play a vital role to ultimately decide whether the physical $D^{0} n n$ system supports a quasi-bound state. With sizable imaginary parts in the meson-neutron scattering lengths, the dynamical effect of the decay channel should be carefully checked in a more robust coupled channel framework. This may be useful to gain better insights into the universal aspects of three-body dynamics, especially in the presence of two-body quasi-bound subsystems with large couplings to open channels. Thus, in regard to drawing definitive conclusions, the present leading order EFT analysis being too simplistic should either be further elaborated by including decay channels (employing complex scattering lengths) followed by sub-leading order effective range corrections, or by employing rigorous few-body techniques combined with realistic two-body potentials, e.g., as pursued in Ref. [29].

\section{Appendix A: Solution via numerical integration}

We present here some details of our methodology used in this work to numerically solve the integral equations (30) and (31) (excluding the three-body force) in order to obtain the eigenenergies, $-E=B_{d}>0$, as a function of the sharp momentum cut-off $\Lambda$. For this purpose, we are only required to solve the homogeneous version of the coupled integral equations written in the general linearized form

$$
\begin{aligned}
& t_{a}=A \otimes t_{a}+B \otimes t_{b}, \\
& t_{b}=C \otimes t_{a}+D \otimes t_{b},
\end{aligned}
$$

or equivalently,

$$
[x]=\mathbf{X} \otimes[x]
$$

with $[x]$, the column eigenvector and $\mathbf{X}$, the transformation matrix, defined as

$$
[x]=\left[\begin{array}{l}
t_{a} \\
t_{b}
\end{array}\right], \quad \mathbf{X}=\left[\begin{array}{ll}
A & B \\
C & D
\end{array}\right]
$$


In the present problem the elements of the $2 \times 2$ transformation matrix $\mathbf{X}\left(p^{\prime}, l\right)$ are given by

$$
\begin{aligned}
& A\left(p^{\prime}, l\right)=\frac{m_{K}}{\pi \mu_{(n K)}} \frac{l^{2} K_{(K)}\left(p^{\prime}, l ; E\right)}{\left[-\frac{1}{a_{d(n K)}}+\sqrt{\frac{\mu_{(n K)}}{\mu_{n(n K)}} l^{2}-2 \mu_{(n K)} E}\right]}, \\
& B\left(p^{\prime}, l\right)=\frac{2 \sqrt{2}}{\pi} \frac{l^{2} K_{(n 1)}\left(p^{\prime}, l ; E\right)}{\left[-\frac{1}{a_{s(n n)}}+\sqrt{\frac{M_{n}}{2 \mu_{K(n n)}} l^{2}-M_{n} E}\right]}, \\
& C\left(p^{\prime}, l\right)=\frac{\sqrt{2} M_{n}}{\pi \mu_{(n K)}} \frac{l^{2} K_{(n 2)}\left(p^{\prime}, l ; E\right)}{\left[-\frac{1}{a_{d(n K)}}+\sqrt{\frac{\mu_{(n K)}}{\mu_{n(n K)}} l^{2}-2 \mu_{(n K)} E}\right]}, \\
& D\left(p^{\prime}, l\right)=0,
\end{aligned}
$$

where the $s$-wave projected one-meson exchange propagator is given as

$$
K_{(K)}\left(p^{\prime}, l ; E\right)=\frac{1}{2 l p^{\prime}} \ln \left[\frac{p^{\prime 2}+l^{2}+a p^{\prime} l-2 \mu_{(n K)} E}{p^{\prime 2}+l^{2}-a p^{\prime} l-2 \mu_{(n K)} E}\right],
$$

while the one-neutron exchange interaction for the elastic and inelastic channels are parametrized by the two propagators:

$$
\begin{aligned}
& K_{(n 1)}\left(p^{\prime}, l ; E\right)=\frac{1}{2 l p^{\prime}} \ln \left[\frac{p^{\prime 2}+b l^{2}+p^{\prime} l-M_{n} E}{p^{\prime 2}+b l^{2}-p^{\prime} l-M_{n} E}\right], \\
& K_{(n 2)}\left(p^{\prime}, l ; E\right)=\frac{1}{2 l p^{\prime}} \ln \left[\frac{b p^{\prime 2}+l^{2}+p^{\prime} l-M_{n} E}{b p^{\prime 2}+l^{2}-p^{\prime} l-M_{n} E}\right] .
\end{aligned}
$$

The integrations are evaluated employing the method of Gaussian quadrature where the integral over the loop momentum variable $l$ is replaced by a summation over $N$ Gauss-Legendre weights $w_{j}$, and correspondingly choosing $N \times N$ intermediate mesh points $\left(p_{i}^{\prime}, l_{j}\right)$ between the integration limits $[0, \Lambda]$, i.e.,

$$
\otimes \equiv \int_{0}^{\Lambda} d l \rightarrow \sum_{j=0}^{N} w_{j} .
$$

To achieve good numerical convergence with a relatively small number of quadrature points, say, $N=96$, we find it convenient to reparametrize the integration variable in the following way: First we assume that the momenta $l, \Lambda$ are re-scaled to dimensionless numbers by removing the dimensional parts, i.e., $l=\tilde{l}[\mu], \Lambda=\tilde{\Lambda}[\mu]$, where $[\mu]$ has unit magnitude carrying the dimension of momentum, i.e., mass dimension 1, while $\tilde{l}, \tilde{\Lambda}$ are dimensionless numbers. Now, we may change the integration variable from $\tilde{l} \rightarrow \zeta$ as

$$
\tilde{l}=\frac{\zeta}{(1-\zeta)^{7}}, \quad d \tilde{l}=d \zeta \frac{1+6 \zeta}{(1-\zeta)^{8}}
$$

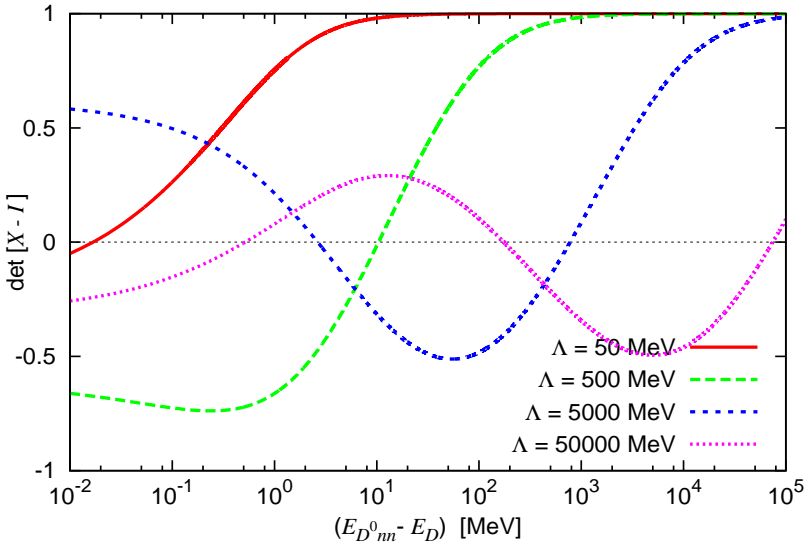

FIG. 12. Det $[X-I]$ vs energy, $-E=\left(E_{D^{0} n n}-E_{D}\right)$, plotted for the $D^{0} n n$ system for different sharp momentum cut-offs $\Lambda$. $E_{D}=1.82 \mathrm{MeV}$ is the threshold energy for break-up into a $D^{0} n$ dimer and a neutron, obtained using the $D^{0} n n$ scattering length in Eq. (36), as the input. The nodes (zeros) correspond to eigenenergies of the trimer level states. Here solutions up to the first three bound states are displayed.

so that the integral equations are reduced to a coupled system of $2 N$ linear algebraic equations for $i=1, \cdots, N$ :

$$
\begin{aligned}
{\left[\begin{array}{c}
t_{a}\left(p_{i}^{\prime}, p ; E\right) \\
t_{b}\left(p_{i}^{\prime}, P ; E\right)
\end{array}\right]=} & \sum_{j=1}^{N} \tilde{w}_{j} \frac{1+6 \zeta_{j}}{\left(1-\zeta_{j}\right)^{8}} \\
& \times\left[\begin{array}{ll}
A\left(p_{i}^{\prime}, \zeta_{j}\right) & B\left(p_{i}^{\prime}, \zeta_{j}\right) \\
C\left(p_{i}^{\prime}, \zeta_{j}\right) & D\left(p_{i}^{\prime}, \zeta_{j}\right)
\end{array}\right]\left[\begin{array}{c}
t_{a}\left(\zeta_{j}, p ; E\right) \\
t_{b}\left(\zeta_{j}, p ; E\right)
\end{array}\right],
\end{aligned}
$$

for a given initial center-of-mass kinetic energy $E$. The new Gaussian weights $\tilde{w}_{j}$ corresponding to $N$ intermediate points $\zeta_{j}$ between the new integration limits $[0, \lambda]$ are related to the previously defined ones by

$$
w_{j}=\tilde{w}_{j} \frac{1+6 \zeta_{j}}{\left(1-\zeta_{j}\right)^{8}}, \quad \tilde{\Lambda}=\frac{\lambda}{(1-\lambda)^{7}} .
$$

On integration, we restore the original form of the momentum cut-off from $\lambda \rightarrow \Lambda$. In this way we may expand the domain of numerical integration to include momenta up to 12 orders of magnitude, maintaining sufficient numerical accuracy.

The consistency of the above system of linear equations imply $\operatorname{det}(X-I)=0$, the solution (zeros) to which determines the eigenenergies for the two-body inputs, namely, the $s$-wave meson-neutron and neutron-neutron scattering lengths. As a demonstration, Fig. 12 displays the plot of $\operatorname{det}[X-I]$ vs energy $-E=\left(E_{D^{0} n n}-E_{D}\right)>0$ for the $D^{0} n n$ system for different sharp cut-offs $(\Lambda)$, using the $D^{0} n$ scattering length in the ZCL [see Eq. (36)] 10

10 It is also clearly revealed in Fig. 10 that trimer eigenenergies $B_{T}$ exist well below $\Lambda \lesssim 100 \mathrm{MeV}$ for the $D^{0} n n$ system. 

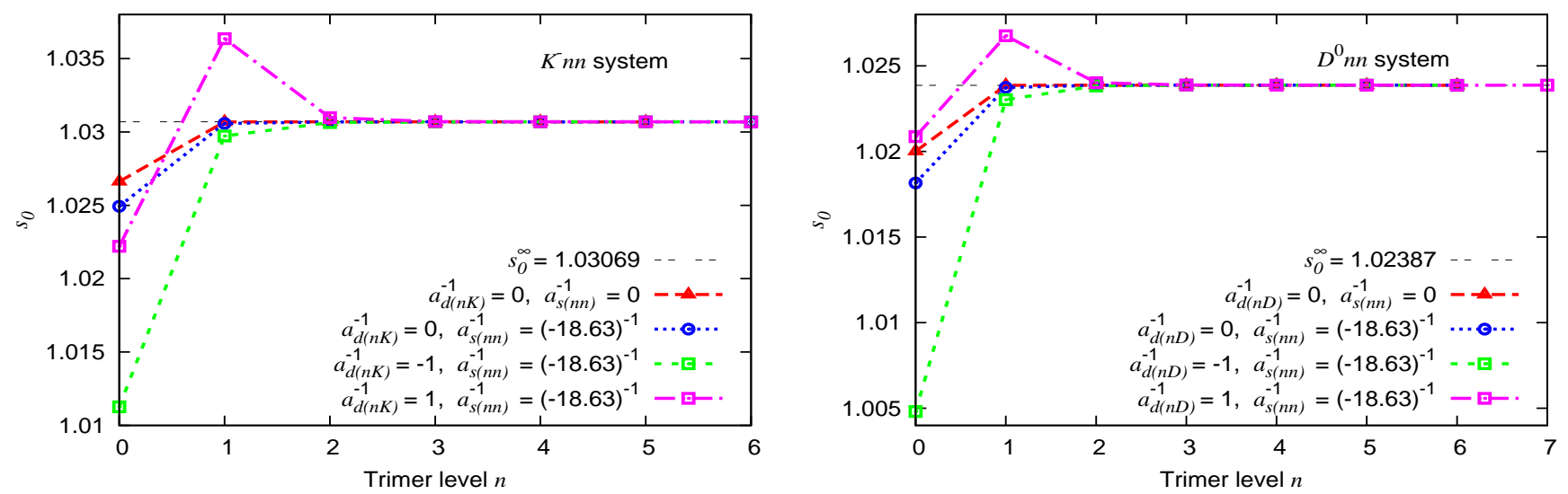

FIG. 13. Convergence of the three-body parameter $s_{0}$ for a fixed eigenenergy, $B_{d}=B_{T}=0.1 \mathrm{MeV}$, for the $K^{-} n n$ and $D^{0} n n$ trimers. The points corresponding to equal values of the two-body (inverse) scattering lengths (in fm $\left.{ }^{-1}\right)$ are connected by straight lines in order to guide the eyes. The long-dashed (red) lines that correspond to true two-body unitarity shows the quickest asymptotic convergence. The other lines correspond to varying the meson-neutron scattering lengths with the neutron-neutron scattering length fixed at the physical value, $a_{s(n n)}=-18.63 \mathrm{fm}$. To achieve reasonable convergence up to the $n=7$ th excited state, the number of quadrature points used is $N=300$.

The figure depicts the kinematic sector with $E_{D^{0} n n}$ above the particle-dimer break-up threshold energy, $E_{D} \simeq$ $1.82 \mathrm{MeV}$, as given by Eq. (40). Below this threshold, i.e., $E_{D^{0} n n}<E_{D}$, the three-body system energetically favors dissociating into a $D^{0} n$ dimer and a neutron. The trimer level states emerge above this threshold which correspond to the zeros of the individual oscillatory curves.

\section{Appendix B: Convergence test of numerical integration}

As a simple qualitative test of the asymptotic convergence of the limit cycle behavior, due to both cut-off dependent artifacts, as well as instabilities arising from the numerical integration, it may be instructive to investigate the behavior of the three-body parameter $s_{0} \equiv s_{0}^{(n)}$ in the non-asymptotic solution. Note the implicit dependence of this parameter on the $n$th critical cut-off, indicated by the superscript $n$. Referring back to Fig. 10 in the main text, if one continues to very large values of the cut-off $\Lambda$, a discrete sequence of critical points is obtained following a rough logarithmic periodicity $\propto \pi / s_{0}^{(n)}$. However, the crucial observation is that as $\Lambda \rightarrow \infty$ this ratio must eventually converge to the universal value $\pi / s_{0}^{\infty}$, where $s_{0}^{\infty}$ is a transcendental number characterizing the asymptotic limit cycle, independent of the scattering lengths and energy eigenvalues of the bound state. In other words, if $\Lambda_{c}^{(n)}$ and $\Lambda_{c}^{(n+1)}$ are successive critical points corresponding to the $n$th and $(n+1)$ th level states $(n=0$ being the ground state) emerging at threshold then,

$$
s_{0}^{(n)}=\frac{\pi}{\ln \left(\frac{\Lambda_{c}^{(n+1)}}{\Lambda_{c}^{(n)}}\right)}, \quad \lim _{n \rightarrow \infty} s_{0}^{(n)}=s_{0}^{\infty} .
$$

This behavior is plotted in Fig. 13 for the case of a possible $K^{-} n n$ bound Borromean state (left plot) and a $D^{0} n n$ bound state (right plot), with fixed energy eigenvalue chosen sufficiently close to the respective thresholds. For the first few values of $n$, the ratios of the critical cut-offs display significant deviations due to finite cut-off effects. In fact, it is to be noted that the ground $(n=0)$ state of three-body systems is predominantly known to display maximal deviations from universal character of the Efimov spectrum, lying far beyond the universal window around the unitary limit. However, both plots suggest a rapid convergence of $s_{0}$ towards the corresponding asymptotic values, $s_{0}^{\infty}=1.03069$ and 1.02387, for $n \geq 2$. This is evident in all cases where different positive and negative two-body (inverse) scattering lengths are chosen approaching the unitary limit from either direction, with the meson masses kept fixed to the respective physical values. In particular, the long-dashed (red) lines in the figure that correspond to true unitarity of the two-body sub-systems show the quickest asymptotic convergence. But despite this apparent convergence, beyond a certain number $n$ the numerical precision of the integration routine becomes poor, resulting in deviations again from the asymptotic values. In that case, numerical accuracy is systematically improved by an appropriate choice of a large value of the number of quadrature points $N$, e.g., $N \geq 300$ may be necessary to obtain reasonable convergence above the $n=7$ th excited Efimov state in the above figure, where the corresponding critical cut-off exceeds $\Lambda_{c}^{(n>7)} \gtrsim 10^{13} \mathrm{MeV}$. But the practical disadvantage of choosing a large $N$ is the dramatic increase in the computational time which is undesirable. However, in the context of our low-energy EFT analysis the numerical integration is naturally restricted to low momentum 
cut-off values where an optimal choice, e.g., $N=96$, yields reasonably good convergence up to the $n=5$ th excited state through the additional reparametrization of the integration variable, as described in Appendix $\mathrm{A}$.

\section{ACKNOWLEDGMENTS}

The authors are grateful to W. Weise, U. van Kolck, and G. Meher for useful discussions. U.R. gratefully acknowledges the hospitality of YITP during the initial stages of the work. S.I.A. acknowledges the hospitality of IIT Guwahati during the final stage of the work. The work of Y.K. was supported in part by JSPS KAKENHI Grant No. JP17J04333. The work of S.I.A. was supported by the Basic Science Research Program through the National Research Foundation of Korea funded by the Ministry of Education of Korea (Grant No. NRF-2016R1D1A1B03930122). The work of T.H. was supported in part by JSPS KAKENHI Grant No. JP16K17694 and by the Yukawa International Program for Quark-Hadron Sciences (YIPQS).
[1] T. Hyodo and D. Jido, Prog. Part. Nucl. Phys. 67, 55 (2012).

[2] A. Gal, E. V. Hungerford and D. J. Millener, Rev. Mod. Phys. 88, 035004 (2016).

[3] Y. Nogami, Phys. Lett. 7, 288 (1963).

[4] Y. Akaishi and T. Yamazaki, Phys. Rev. C 65, 044005 (2002).

[5] N. V. Shevchenko, A. Gal and J. Mares, Phys. Rev. Lett. 98, 082301 (2007).

[6] N. V. Shevchenko, A. Gal, J. Mares and J. Revai, Phys. Rev. C 76, 044004 (2007),.

[7] Y. Ikeda and T. Sato, Phys. Rev. C 76, 035203 (2007).

[8] Y. Ikeda and T. Sato, Phys. Rev. C 79, 035201 (2009).

[9] T. Yamazaki and Y. Akaishi, Phys. Rev. C 76, 045201 (2007).

[10] A. Dote, T. Hyodo and W. Weise, Nucl. Phys. A 804, 197 (2008).

[11] A. Dote, T. Hyodo and W. Weise, Phys. Rev. C 79, 014003 (2009).

[12] S. Wycech and A. Green, Phys. Rev. C 79, 014001 (2009).

[13] Y. Ikeda, H. Kamano and T. Sato, Prog. Theor. Phys. 124, 533 (2010).

[14] N. Barnea, A. Gal and E. Liverts, Phys. Lett. B 712, 132 (2012).

[15] S. Ohnishi, W. Horiuchi, T. Hoshino, K. Miyahara and T. Hyodo, Phys. Rev. C 95, 065202 (2017).

[16] J. Revai, Phys. Rev. C 94, 054001 (2016)

[17] T. Hoshino, S. Ohnishi, W. Horiuchi, T. Hyodo and W. Weise, Phys. Rev. C 96, 045204 (2017).

[18] T. Uchino, T. Hyodo and M. Oka, Nucl. Phys. A 868869, 53 (2011).

[19] A. Hosaka, T. Hyodo, K. Sudoh, Y. Yamaguchi and S. Yasui, Prog. Part. Nucl. Phys. 96, 88 (2017).

[20] N. Brambilla et al., Eur. Phys. J. C 71, 1534 (2011).

[21] A. Hosaka, T. Iijima, K. Miyabayashi, Y. Sakai and S. Yasui, PTEP 2016, 062C01 (2016).

[22] C. Patrignani, Chin. Phys. C40, 100001 (2016).

[23] J. Hofmann and M. F. M. Lutz, Nucl. Phys. A 763, 90 (2005).

[24] T. Mizutani and A. Ramos, Phys. Rev. C 74, 065201 (2006).

[25] C. Garcia-Recio et al., Phys. Rev. D 79, 054004 (2009).

[26] J. Haidenbauer, G. Krein, U.-G. Meissner and L. Tolos, Eur. Phys. J. A 47, 18 (2011).
[27] Y. Yamaguchi, S. Ohkoda, S. Yasui and A. Hosaka, Phys. Rev. D 84, 014032 (2011).

[28] Y. Yamaguchi, S. Ohkoda, S. Yasui and A. Hosaka, Phys. Rev. D 87, 074019 (2013).

[29] M. Bayar et al., Phys. Rev. C 86, 044004 (2012).

[30] Y. Yamaguchi, S. Yasui and A. Hosaka, Nucl. Phys. A 927, 110 (2014).

[31] E. Braaten and H.-W. Hammer, Phys. Rept. 428, 259 (2006).

[32] P. Naidon and S. Endo, Rept. Prog. Phys. 80, 056001 (2017).

[33] V. Efimov, Phys. Lett. B 33, 563 (1970).

[34] E. Braaten and H. Hammer, Phys. Rev. Lett. 91, 102002 (2003).

[35] S. König, H. W. Grießhammer, H. W. Hammer and U. van Kolck, Phys. Rev. Lett. 118, no. 20, 202501 (2017).

[36] D. L. Canham, H. W. Hammer, and R. Springer, Phys. Rev. D 80, 014009 (2014).

[37] E. Braaten and M. Kusunoki, Phys. Rev. D 69, 074005 (2004).

[38] T. Hyodo, T. Hatsuda and Y. Nishida, Phys. Rev. C 89, 032201 (2014).

[39] S.-I. Ando, U. Raha and Y. Oh, Phys. Rev. C 92, 024325 (2015).

[40] N. Barnea, L. Contessi, D. Gazit, F. Pederiva and U. van Kolck, Phys. Rev. Lett. 114, no. 5, 052501 (2015).

[41] M. Bazzi et al. (SIDDHARTA Collaboration), Phys. Lett. B 704, 113 (2011).

[42] M. Bazzi et al. (SIDDHARTA Collaboration), Nucl. Phys. A 881, 88 (2012).

[43] U. G. Meissner, U. Raha and A. Rusetsky, Eur. Phys. J. C 35, 349 (2004).

[44] Y. Ikeda, T. Hyodo and W. Weise, Phys. Lett. B 706, $63(2011)$.

[45] Y. Ikeda, T. Hyodo and W. Weise, Nucl. Phys. A 881, 98 (2012).

[46] Y. Yamaguchi and T. Hyodo, Phys. Rev. C 94, 065207 (2016).

[47] N. Kaiser, P. B. Siegel and W. Weise, Nucl. Phys. A 594, 325 (1995).

[48] E. Oset and A. Ramos, Nucl. Phys. A 635, 99 (1998).

[49] J. A. Oller and U. G. Meissner, Phys. Lett. B 500, 263 (2001).

[50] T. Hyodo, D. Jido and A. Hosaka, Phys. Rev. C 78, 025203 (2008). 
[51] T. Hyodo and W. Weise, Phys. Rev. C 77, 035204 (2008).

[52] R. Eden and J. Taylor, Phys. Rev. 133, B1575 (1964).

[53] B. C. Pearce and B. F. Gibson, Phys. Rev. C 40, 902 (1989).

[54] A. Cieply, M. Mai, U.-G. Meissner and J. Smejkal, Nucl. Phys. A 954, 17 (2016).

[55] T. Hyodo, Phys. Rev. C 90, 055208 (2014).

[56] Y. Kamiya and T. Hyodo, Phys. Rev. D 97, 054019 (2018).

[57] H. W. Hammer, Nucl. Phys. A 705, 173 (2002).

[58] S.-I. Ando and M. C. Birse, J. Phys. G 37, 105108 (2010).

[59] S.-I. Ando, G.-S. Yang, and Y. Oh, Phys. Rev. C 89, 014318 (2014).

[60] S.-I. Ando and Y. Oh, Phys. Rev. C 90, 037301 (2014).

[61] H. W. Hammer, C. Ji, and D. R. Phillips, J. Phys G 44, 103002 (2017)

[62] D. B. Kaplan, M. J. Savage and M. B. Wise, Phys. Lett. B 424, 390 (1998).

[63] D. B. Kaplan, M. J. Savage and M. B. Wise, Nucl. Phys. B 534, 329 (1998).
[64] U. van Kolck, Nucl. Phys. A 645, 273-302 (1999).

[65] S. R. Beane and M. J. Savage, Nucl. Phys. A 694, 511 (2001).

[66] S.-I. Ando and C. H. Hyun, Phys. Rev. C 72, 014008 (2005).

[67] P. F. Bedaque, H. W. Hammer, and U. van Kolck, Nucl. Phys. A 646, 444 (1999).

[68] G. V. Skornyakov and K. A. Ter-Martirosyan, Sov. Phys. JETP 4, 648 (1957) [Zh. Eksp. Teor. Fiz. 31, 775 (1956)].

[69] G. V. Skornyakov and K. A. Ter-Martirosyan, JETP 31, 775 (1956).

[70] D. L. Canham and H. W. Hammer, Eur. Phys. J. A 37, 367 (2008).

[71] G. S. Danilov and V. I. Lebedev, Sov. Phys. JETP 17 (1963) 1015.

[72] Q. Chen et al., Phys. Rev. C 77, (2008), 054002-1. 